\title{
Biomedical photoacoustics: fundamentals, instrumentation and perspectives on nanomedicine
}

\author{
This article was published in the following Dove Press journal: \\ International Journal of Nanomedicine \\ 22 December 2016 \\ Number of times this article has been viewed
}

\author{
Chunpeng Zou, ',* Beibei \\ Wu,' Yanyan Dong,' \\ Zhangwei Song,' Yaping \\ Zhao, ',* Xianwei Ni,' Yan \\ Yang,' Zhe Liu'l-3 \\ 'Department of Ultrasonic Diagnosis, \\ The Second Affiliated Hospital and \\ Yuying Children's Hospital, Wenzhou \\ Medical University, ${ }^{2}$ Wenzhou \\ Institute of Biomaterials and \\ Engineering, Chinese Academy \\ of Sciences, ${ }^{3}$ Wenzhou Institute \\ of Biomaterials and Engineering, \\ Wenzhou Medical University, \\ Wenzhou, People's Republic of China \\ *These authors contributed equally \\ to this work
}

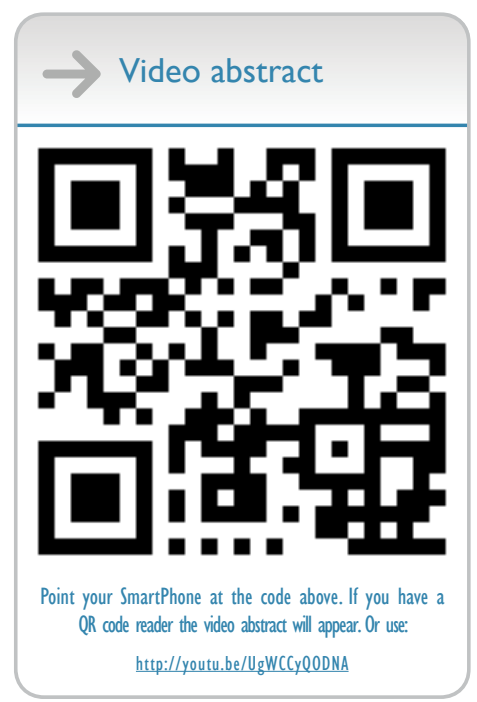

Correspondence: Zhe Liu; Yan Yang Department of Ultrasonic Diagnosis, The Second Affiliated Hospital and Yuying Children's Hospital, Wenzhou Medical University, Wenzhou 325027, People's Republic of China Email liuzhe@wibe.ac.cn; yang25yan@I26.com

\begin{abstract}
Photoacoustic imaging (PAI) is an integrated biomedical imaging modality which combines the advantages of acoustic deep penetration and optical high sensitivity. It can provide functional and structural images with satisfactory resolution and contrast which could provide abundant pathological information for disease-oriented diagnosis. Therefore, it has found vast applications so far and become a powerful tool of precision nanomedicine. However, the investigation of PAI-based imaging nanomaterials is still in its infancy. This perspective article aims to summarize the developments in photoacoustic technologies and instrumentations in the past years, and more importantly, present a bright outlook for advanced PAI-based imaging nanomaterials as well as their emerging biomedical applications in nanomedicine. Current challenges and bottleneck issues have also been discussed and elucidated in this article to bring them to the attention of the readership.
\end{abstract}

Keywords: photoacoustics, imaging, therapy, nanomaterials, nanomedicine

\section{Introduction \\ Principle of photoacoustics}

Photoacoustics is an emerging diagnostic imaging strategy which helps to obtain three-dimensional (3D) tomographic information of tissues or lesions. ${ }^{1-3}$ The irradiation of a pulsed laser gives rise to local heating and energy absorbance of photosensitive agents, which causes expansion of absorber. This expansion will squeeze the surrounding tissues to produce acoustic waves which are transmitted to the transducer for image reconstruction (Figure 1), and this is named as photoacoustic (PA) effect. PA effect is based on the difference in the acoustic waves caused due to difference in light absorption rates in different organisms, which makes it possible to distinguish the different structures of the test object. ${ }^{2,3}$ In 1880, Bell observed this phenomenon, and in the late 1990s, photoacoustic imaging (PAI) was developed as a brand-new biomedical imaging modality. ${ }^{4}$

Since PAI integrates the advantages of acoustic deep penetration and optical high sensitivity, it is able to provide images with high resolution and detailed structural information for biological and pathological processes. As a result, PAI has seen broad biomedical applications especially in cancer detection, atherosclerotic plaque mapping, brain and retinal imaging and even PAI-guided treatments. ${ }^{5-8}$ With the rapid development and upgrading of PAI technology, the ever-growing requirements in the quality and performance of PAI are believed to be met in the coming decades.

\section{Instrumentations for PAI}

Based on an ultrasound (US) imaging system, VisualSonics developed Vevo LAZR photoacoustic platform for small-animal preclinical imaging. Ease of control and 


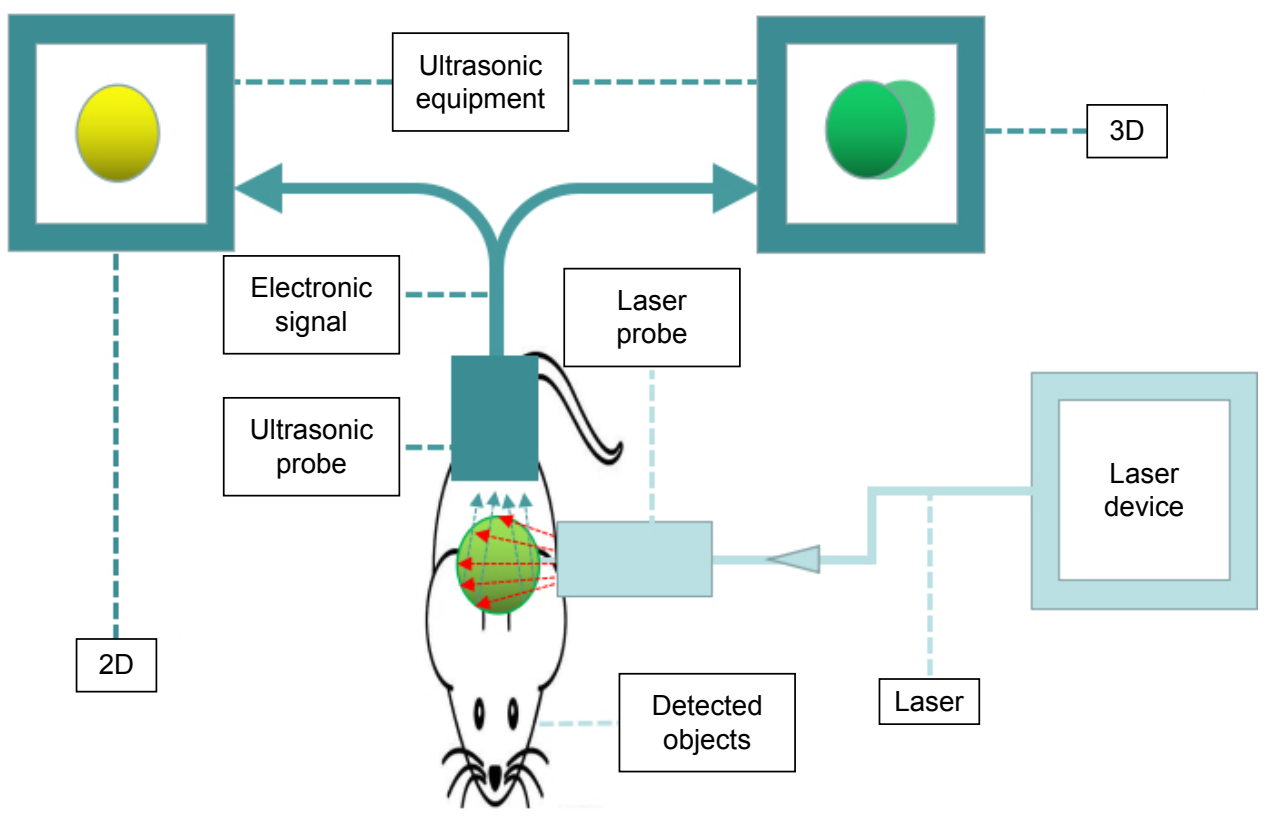

Figure I Illustration for the nature and principle of photoacoustics. Abbreviations: 3D, three-dimensional; 2D, two-dimensional.

whole-body imaging are the apparent advantages of in vivo real-time imaging. Endra Life Sciences also manufactured Nexus 128 for small-animal PAI of cancers for preclinical uses. Advanced two-dimensional (2D) and 3D images may be acquired through a built-in analysis software package in this system which generates high-resolution PAI pictures. By correcting temperature changes, Van de Sompel et al realized improved image quality in both phantom and tissues with Endra Nexus $128 .{ }^{9}$ Besides that, iThera Medical and Seno Medical Instruments have also developed PAI instruments MSOT (multi-spectral optoacoustic tomography) and LOUIS-3D, respectively. Aiming at clinical application, the MSOT Acuity was developed with a fast-tunable $50 \mathrm{~Hz}$ laser and 2D and 3D detectors by iThera Medical, which has been proven to achieve the real-time biomedical imaging and quantitative imaging of lesions through the tissue chromophore distribution and concentration. Comparing both detectors, the 2D probe performs better in lowering signal decrease and anatomical resemblance, while the $3 \mathrm{D}$ probe is prominent in rapid $3 \mathrm{D}$ imaging which enables the visualization of superficial areas. ${ }^{10}$ In addition, the Twente Group in collaboration with ESAOTE Europe BV developed a dual-imaging modality by combing a US transducer array and a diode stack laser in a single US probe connected to a commercially available US system. The new modality integrates the advantages of both US and PAI in imaging, with the anatomic details being provided by US and the functional information by PAI. ${ }^{11}$ It should be noted that PA scanners have been applied in clinics as a prototype for augmenting the US techniques such as sentinel lymph node (SLN) dye injection (Table 1).

Table I Typical commercial PAI instrumentations for preclinical and clinical studies

\begin{tabular}{|c|c|c|c|}
\hline Instrument & Modality & Advantage & Application \\
\hline LAZR (VisualSonics) $)^{12,13}$ & $\begin{array}{l}\text { Ultrasound-photoacoustic } \\
\text { imaging integrated }\end{array}$ & $\begin{array}{l}\text { Whole-body with sectional PAI } \\
\text { options }\end{array}$ & Preclinical \\
\hline Nexus I 28 (Endra Life Sciences) ${ }^{14}$ & Photoacoustic imaging & $\begin{array}{l}\text { Fast imaging and high-resolution } \\
3 \mathrm{D} \text { image reconstruction }\end{array}$ & Preclinical cancer detection \\
\hline MSOT (iThera Medical) ${ }^{10,15}$ & Photoacoustic tomography & $\begin{array}{l}\text { Real-time, whole-body } \\
\text { tomography with body navigations }\end{array}$ & $\begin{array}{l}\text { Preclinical whole-body PAI especially } \\
\text { cancer detection and brain imaging }\end{array}$ \\
\hline $\begin{array}{l}\text { LOUIS-3D (Tomo Wave } \\
\text { Laboratories, Inc.) })^{16}\end{array}$ & $\begin{array}{l}\text { Photoacoustic imaging } \\
\text { with tunable laser switch }\end{array}$ & $\begin{array}{l}\text { The first PAI scanner for SLN dye } \\
\text { injection guidance }\end{array}$ & $\begin{array}{l}\text { Preclinical and clinical studies on } \\
\text { oncology, vascular angiography, } \\
\text { hematology, etc. }\end{array}$ \\
\hline
\end{tabular}

Abbreviations: PAI, photoacoustic imaging; 3D, three-dimensional; SLN, sentinel lymph node. 


\section{Biomedical photoacoustics and sub-modalities of PAI}

Biomedical photoacoustics offers real-time, noninvasive imaging with a penetration depth up to $5 \mathrm{~cm} .{ }^{17}$ On the other hand, PAI is naturally suitable for molecular imaging which not only provides structural information of tissues but also offers information on physiological functional signs such as oxygen saturation, hemoglobin concentration and angiogenesis. ${ }^{18-23}$ So far, PAI has been combined with other imaging techniques such as multiphoton microscopy (MPM), optical coherence tomography (OCT) and fluorescence (FL), to form a family of several prevailing sub-modalities including PA computed tomography (PACT), PA microscopy (PAM), PA endoscopy (PAE), PA flow cytometry (PAFC) and magnetomotive PAI (mmPAI) as described in Table 2.

Based on the computed image reconstruction, PACT can provide deeper penetration and larger imaging scope due to the signal generation from diffused photons. Moreover, the imaging scale is changeable to meet specific applications. The present biomedical translation of PACT consists of tumor imaging, SLN imaging and superficial and deep vessel mapping. ${ }^{24-27}$ In addition, the multispectral PACT multivariate curve resolution alternating least squares has been recently applied to visualize the peripheral nerves. ${ }^{28}$ PA Doppler flowmetry is another application of PACT which implements PA Doppler effect to image blood flow in vivo with high spatial resolution and sensitivity.

In contrast to PACT, PAM detects PA signals generated from the transducer focus, and thus, it does not require inverse reconstruction algorithm. According to the pathway to achieve lateral resolution, acoustic-resolution PAM (AR-PAM) and optical-resolution PAM (OR-PAM) have

Table 2 Medical sub-modalities of PAI

\begin{tabular}{|c|c|c|c|}
\hline Modality & Application & Advantage & Status \\
\hline PACT & $\begin{array}{l}\text { Peripheral joints, } \\
\text { brain, whole-body } \\
\text { study }\end{array}$ & $\begin{array}{l}\text { Real-time and } \\
\text { tomographic imaging }\end{array}$ & $\begin{array}{l}\text { Preclinical } \\
\text { and clinical }\end{array}$ \\
\hline PAM & $\begin{array}{l}\text { Molecular or } \\
\text { cellular imaging }\end{array}$ & $\begin{array}{l}\text { Microcirculation } \\
\text { imaging without } \\
\text { exogenous contrast }\end{array}$ & Preclinical \\
\hline PAE & $\begin{array}{l}\text { Gastrointestinal } \\
\text { or cardiovascular } \\
\text { imaging }\end{array}$ & $\begin{array}{l}\text { Gastrointestinal tract } \\
\text { imaging }\end{array}$ & $\begin{array}{l}\text { Partially } \\
\text { clinical }\end{array}$ \\
\hline PAFC & $\begin{array}{l}\text { Circulating tumor } \\
\text { cells detection }\end{array}$ & $\begin{array}{l}\text { Quantitative flow } \\
\text { cytometry imaging }\end{array}$ & Preclinical \\
\hline mmPAl & $\begin{array}{l}\text { Circulating tumor } \\
\text { cells detection }\end{array}$ & $\begin{array}{l}\text { Specific contrast } \\
\text { enhancement availability }\end{array}$ & Preclinical \\
\hline
\end{tabular}

Abbreviations: PAI, photoacoustic imaging; PACT, photoacoustic computed tomography; PAM, photoacoustic microscopy; PAE, photoacoustic endoscopy; PAFC, photoacoustic flow cytometry; mmPAl, magnetomotive PAI. been differenciated..$^{29,30}$ In AR-PAM, the acoustic focus is usually more concentrated than the optical focus, and thus, the system is offered by acoustic resolution. The lateral resolution in AR-PAM is primarily decided by the ultrasonic focal spot. In OR-PAM, the optical focus is more concentrated than the acoustic focus, and thus, the system is offered by optical resolution. The imaging process is similar to AR-PAM, but both ultrasonic detection and laser illumination are highly focused and arranged in a confocal geometry, which has the lateral resolution determined by the optical focal spot. The spatial resolution of OR-PAM is much higher than that of AR-PAM as a result of shorter optical wavelength down to sub-micrometers. ${ }^{31}$ On the other hand, PAM can display microcirculation of live animals without exogenous contrasts (Figure 2). ${ }^{32}$ Moreover, it offers molecular or genetic functional imaging to accurately measure oxygen saturation and hemoglobin concentration in vivo. ${ }^{33,34}$

In virtue of imaging purposes on the gastrointestinal tract and cardiovascular system, PAE exhibits high imaging speed and has a special ultrasonic transducer. Recently, a US transducer for PAE has been upgraded with a diameter of $3.8 \mathrm{~mm}$ and a length of $38 \mathrm{~mm}$, which enables the simultaneous US and PAI with a single device. ${ }^{3}$ Further technical improvement can be achieved by eliminating motion artifacts and reducing transducer diameter to $1 \mathrm{~mm} .{ }^{35}$ By employing exogenous contrast agents, it is promising to further enhance the image contrast and accuracy of PAE for gastrointestinal tract imaging and earlier detection of premalignant and malignant lesions. In addition, scientists have achieved the percutaneous coronary intervention with the guidance of intravascular PAE in vivo. ${ }^{36}$

The detection of circulating tumor cells (CTCs) always requires quantitative analysis and accurate sorting of detectable cell populations. For this purpose, PAI combined with the flow cytometry (PAFC) is developed. Unlike the traditional flow cytometry, PAFC overcomes the shortcomings of low sensitivity and invasive cell extraction. However, when employing this technique in vivo, several issues must be taken into consideration such as access to deep vessels, blood flow instability and multiple-file cell flow in vessel cross sections.

mmPAI is developed especially to enhance specific contrast in PA molecular imaging by suppressing background signals. ${ }^{37}$ This technique implements the labeling of photoabsorbing nanoparticles and paramagnetic substances, which activates PA detection and magnetic manipulation at the same time. With the mmPAI, CTCs can be differentiated from the blood, whereas traditional PAI is inefficient in detecting 


\section{A}

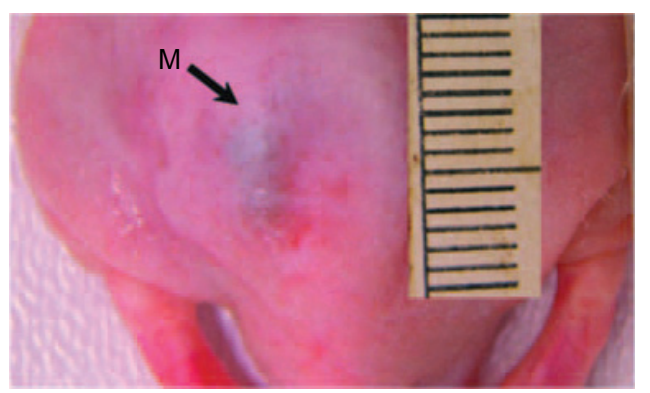

C

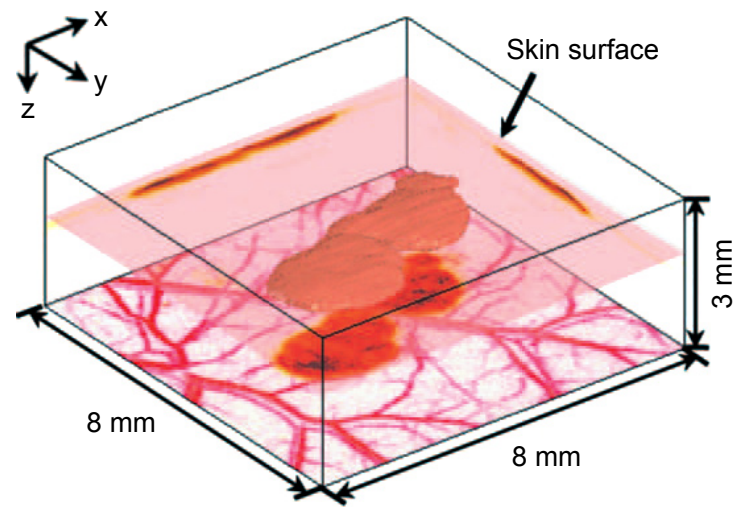

B
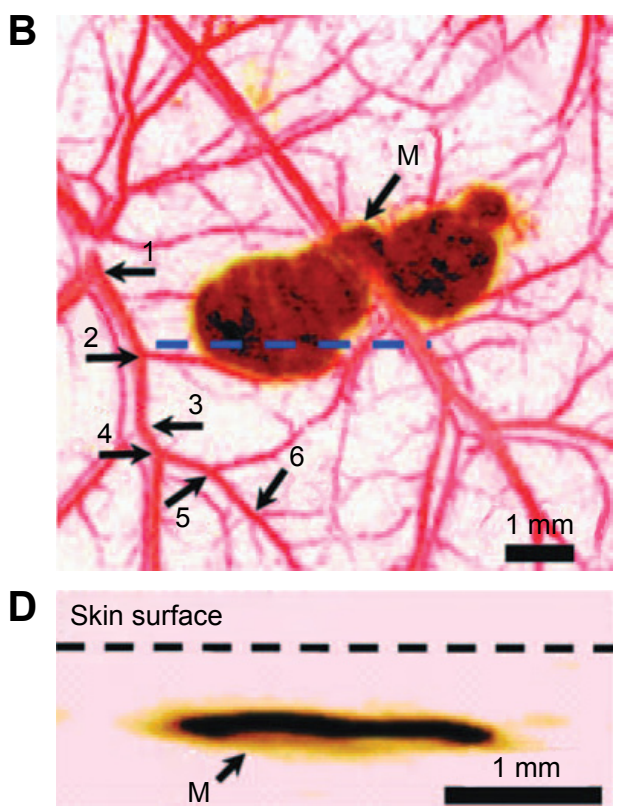

E

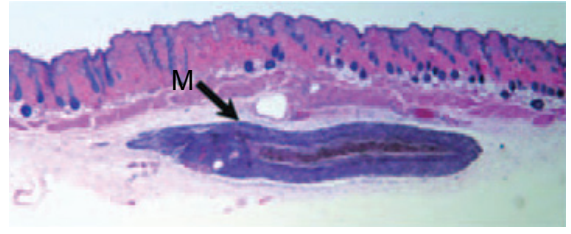

Figure 2 (A) Picture of a melanoma in a nude mouse. (B) PAM image obtained with a 50 MHz PAM system. (C) 3D demonstration of the melanoma. (D) Close-up 2D image of the melanoma. (E) HE-stained histology at the identical marked location. Vessel branching was indicated by numbers I-6. Melanoma is indicated by "M".

Notes: Reprinted with permission from Macmillan Publishers Ltd: Nat Biotechnol. Zhang HF, Maslov K, Stoica G, Wang LV. Functional photoacoustic microscopy for highresolution and noninvasive in vivo imaging. 24(7):848-85I. Copyright 2006. ${ }^{32}$

Abbreviations: PAM, photoacoustic microscopy; 3D, three-dimensional; 2D, two-dimensional; HE, hematoxylin-eosin.

rare CTCs due to the heterogeneous background with strong optical-scattering noises.

Except for the conventional types of PAI, there is a number of hybrid modalities of PAI combined with other imaging techniques. FL imaging is a nonionizing technology employed in molecular imaging. However, due to its strong optical diffusion, FL suffers from inadequate penetration depth and low spatial resolution. To compensate these shortcomings of FL, PAI is integrated with it by injecting a single contrast agent which has a proper FL quantum yield. The combination of these techniques has been applied in the bladder imaging in rats with indocyanine green (ICG) as the contrast. ${ }^{38}$ MPM, based on stimulating fluorescent-labeled molecules, provides more molecular information with less photobleaching and wider wavelength-tuning range than the traditional FL microscopy. Combined with PAM and confocal microscopy, MPM can be extended to the new biological field for measuring non-fluorescent objects such as melanin and hemoglobin. ${ }^{18}$ Furthermore, the combined PAM/OCT system is made up of both PAM and OCT, a 2D galvanometer for raster scanning and a dichroic mirror integrated with the pulsed laser beam in PAM and broadband beam in OCT. ${ }^{39}$
PAI and thermoacoustic tomography (TAT) have been integrated by Ku et al in their laboratory as PAI/TAT system which combines near-infrared (NIR) laser light and nonionizing radiofrequency waves to acquire additional information such as blood volume, oxygenation level of hemoglobin and water/ion concentration from breast tissue. ${ }^{40}$

\section{Advanced nanomaterials for PAl}

The contrast of PA images may be enhanced by employing endogenous and/or exogenous contrast agents. The endogenous contrast agents for PAI such as melanin and hemoglobin have been utilized to evaluate the oxygen saturation and hemoglobin concentration in tumors. ${ }^{41}$ On the other hand, the development of exogenous contrast agents has attracted increasing attention, and numerous advanced nanomaterials including gold nanoparticles (AuNPs), single-walled carbon nanotubes (SWNTs), semi-conductive nanoparticles, organic dyes, etc. have been designed as contrast agents for PA molecular imaging (Figure 3). ${ }^{42-44}$ Issues related to biosafety of these advanced nanomaterials are being focused on so that these nanomaterials can be translated to clinical uses. Apart from that, prominent sensitivity, proper stability and 


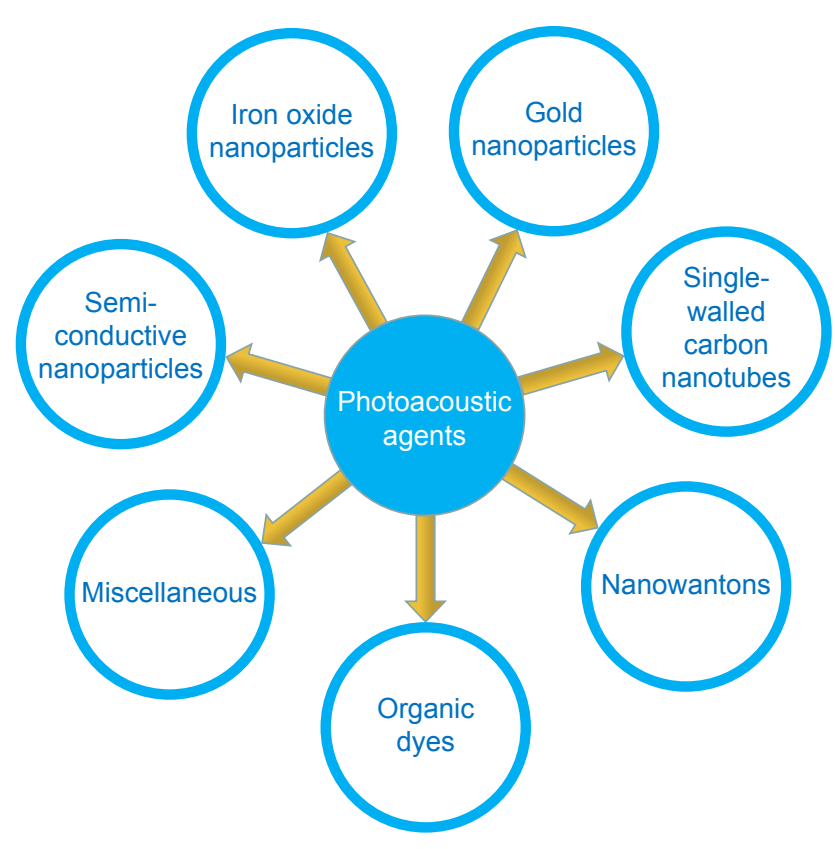

Figure 3 Advanced nanomaterials as exogenous contrast agents for photoacoustic imaging (PAI).

good tolerance to appropriate circulation microenvironment in vivo are prerequisites in terms of nanomedicine and have now become hot research topics.

\section{Gold nanoparticles}

AuNPs can be stimulated by NIR radiation and generate PA waves for PAI. Compared with small-molecule fluorophores, AuNPs are not prone to photobleaching or autofluorescence, and they exhibit low light scattering but strong penetration. ${ }^{45}$ Localized surface plasmon resonance (LSPR) is a general phenomenon exhibited by AuNPs due to their inherent light absorbance and scattering at specific wavelengths, and by alteration of superficial coating or particle shape of AuNPs, LSPR can be manipulated for biomedical imaging.

AuNPs as exogenous contrast agents are able to image vulnerable plaque markers. After endocytosis by macrophages, they aggregate and produce a shift in the absorption peak which is different from that produced by the non-aggregated nanoparticles. Wang et al proved this by injecting AuNPs-loaded macrophages in an atherosclerotic rabbit aorta ${ }^{46}$ Yeager et al also embarked on the intravascular photoacoustic imaging (IVPAI) of the atherosclerotic rabbit aorta with injection of AuNPs, and it revealed the atherosclerotic area with acute inflammations and compromised luminal endothelium by high PA signals from the AuNPs extravasation. ${ }^{47}$

Another sort of AuNPs developed for PAI is gold nanocages (AuNCs) which show characteristic hollow interiors enclosed by a single ultrathin crystal with porous walls. ${ }^{48}$
Skrabalak et $\mathrm{al}^{49}$ and Chen et $\mathrm{al}^{50}$ synthesized a series of AuNCs and demonstrated that with variation of AuNCs size and thickness, their wavelengths in the range of NIR region could be switchable. Besides, AuNCs could be used for targeted drug delivery with satisfactory biocompatibility and less metal toxicity. These superior features make AuNCs qualified for versatile biomedical applications, especially for SLN mapping. In SLN localization, strong imaging contrast and high spatial resolution at a penetration depth of $33 \mathrm{~mm}$ have been achieved (Figure 4). ${ }^{51}$ It overran the methodology of methylene blue and radioactive colloids which had low spatial resolution, low sensitivity and adverse effects.

Gold nanoshells are a sort of AuNPs consisting of silica cores with gold shell coating outside. Similar to AuNCs, the optical absorption of gold nanoshells relies on the rigid metallic structures as well as the variable core sizes and thicknesses. The contrast enhancement of gold nanoshells for PAI in vivo was proven by acquisition of PACT images of Sprague Dawley rats cerebral cortexes injected with PEGylated nanoshells. Owing to the prominent biocompatibility and availability to target malignant tumors, gold nanoshells have been extensively applied in diagnosis of vasculature tumors, photothermal therapy (PPT) and chemotherapy using hollow structures as antitumor drug carriers. ${ }^{52,53}$

The gold nanobeacons are synthesized by entrapping small spherical AuNPs within a colloidal particle and then encapsulating them with a biocompatible phospholipids coating. ${ }^{54}$ The entrapment of small AuNPs is presumed to enhance the signals, while the AuNPs can be metabolized and eliminated through the urinary system. Moreover, the aggregation of small AuNPs will lead to the tunable absorption peaks for best in vivo PAI signals.

The first-generation gold nanobeacons (GNB1) displayed prominent stability in both size and zeta potential. The PA signal of rat blood mixed with GNB1 suspension was much stronger, that is, 10 times higher, than that of pure blood. ${ }^{48}$ Due to this outstanding property, $\alpha v \beta 3$-integrin overexpressed on endothelial cells during non-polarized angiogenesis was integrated with GNB1 for imaging neovascularization. The first case of neovascularization was implemented and succeeded in demonstrating the process of angiogenesis including nascent neovessel tubules, sprouts and bridges. With the injection of $\alpha v \beta 3$-targeted rhodaminelabeled GNB1, angiogenesis could be discriminated from the microvasculature with high sensitivity and specificity. ${ }^{54}$

\section{Single-walled carbon nanotubes}

The strong optical properties make the carbon nanotubes applicable to PAI, whereas the lower NIR absorption compared 

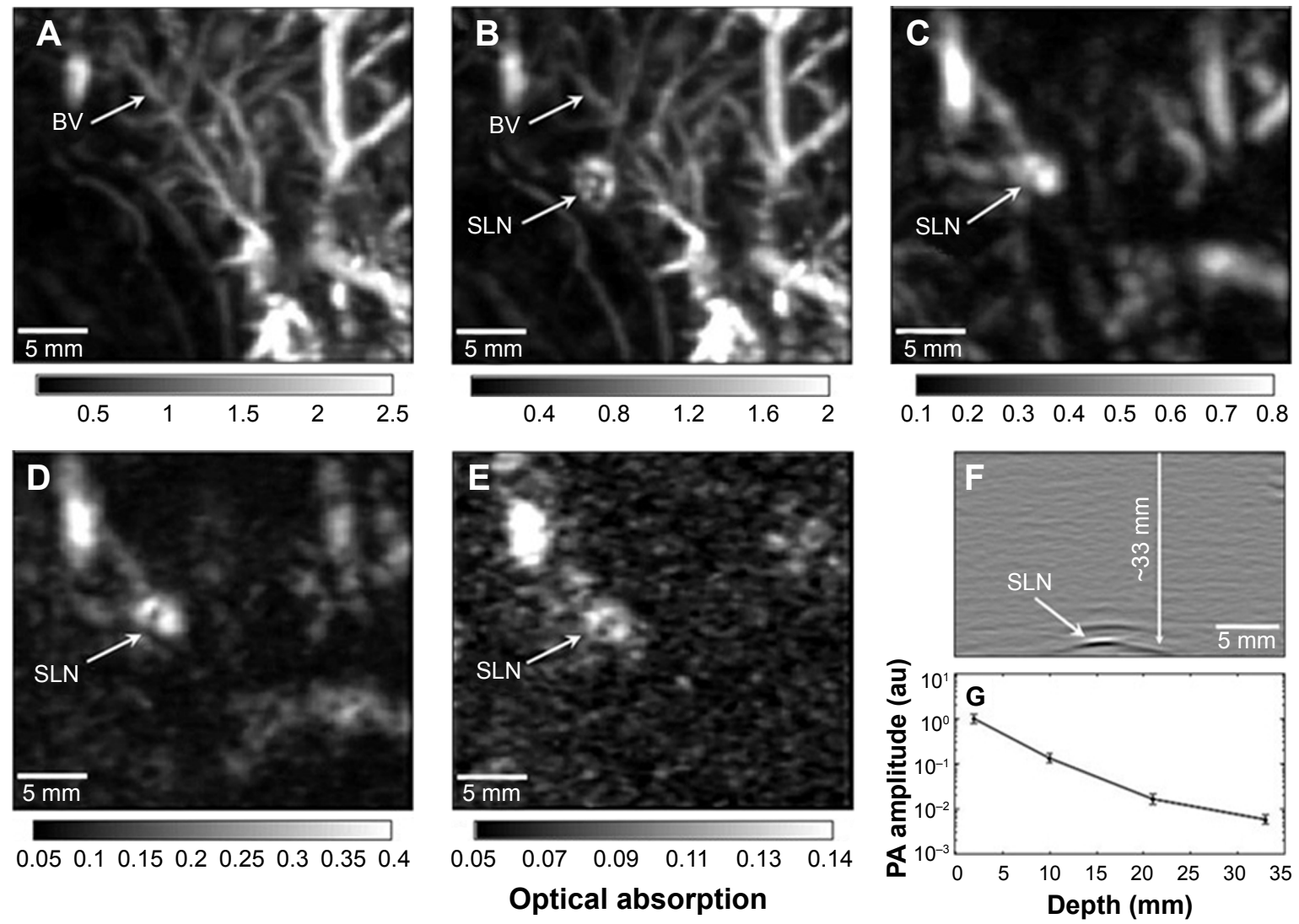

Figure 4 Photoacoustic sagittal maximum amplitude projection images before $(\mathbf{A})$ and after (B-E) the injection: (A) control image; (B) 28 min; (C) I26 min with a layer of chicken breast tissue placed on axillary region, total imaging depth was $10 \mathrm{~mm}$; (D) $165 \mathrm{~min}$ with the second layer of chicken breast tissue, total imaging depth was 21 mm; (E) $226 \mathrm{~min}$ with the third layer of chicken breast tissue, total imaging depth was $33 \mathrm{~mm}$. (F) Photoacoustic B-scan with 20 times signal average, showing the SLN located $33 \mathrm{~mm}$ deep. Memory of the acquisition system limited the record length in depth. (G) The amplitude variations of photoacoustic signals over imaging depths. Data were scaled down to $0 \mathrm{~dB}$ and normalized by the maximum. Error bar represents standard deviation. All images were acquired without signal averaging except the B-scan (F). Color bars represent the optical absorption.

Notes: Reprinted with permission from Song KH, Kim C, Cobley CM, Xia Y, Wang LV. Near-infrared gold nanocages as a new class of tracers for photoacoustic sentinel lymph node mapping on a rat model. Nano Lett. 2009;9(I):183-188..$^{11}$ Copyright 2009 American Chemical Society.

Abbreviations: PA, photoacoustic; BV, blood vessel; SLN, sentinel lymph node.

with AuNPs prevents further enhancement of PA signals. In this respect, SWNTs address the issue by more exposure to optical radiation due to a high surface area.$^{55}$ To further boost the optical absorption, a hybrid Au-SWNT was fabricated with the SWNTs core coated by a gold layer. It facilitated the biocompatible bio-conjugation of drug molecules. Apart from that, organic dyes can be coupled onto the surface of SWNTs to augment the optical absorption in NIR region as well. This has been validated by loading ICG molecules onto SWNTs which gains a 20 -fold increase in NIR absorption. ${ }^{56}$

Integrin-targeted SWNTs were synthesized by covalently conjugating SWNTs with arginine-glycine-aspartate (RGD) peptides for neovascularization imaging. Higher PA signals in the tumor region were observed than the non-conjugated control (Figure 5) ${ }^{44}$ Although the toxicity of SWNTs is still under investigation, a number of studies have disclosed their applications in PAI as promising contrast agents for tumor neovasculature imaging, SLN mapping as well as PPT.

\section{Semi-conductive nanoparticles}

Semi-conductive polymer nanoparticles (SPNs) are an emerging type of PAI nanomaterials, and have displayed excellent properties such as non-blinking, low toxicity, prominent FL and excellent photostability. ${ }^{57}$ Therefore, with a good control of surface chemistry and versatile modifications of functional groups, enormous SPNs have emerged as contrast agents for cell labeling and PAI. ${ }^{58}$ SPNs can be further developed into NIR ratiometric nanoprobes for in vivo imaging of reaction oxygen species (ROS). Considering that ROS acts as a specific biomarker in many pathological processes, SPNs-based nanomaterials are promisingly utilized in the study of strokes, tumor metastasis, bacterial infections and cardiomyopathy.

Quantum dots are another kind of semi-conductive nanocrystals with tiny dimensions but wide excitation spectra. They have exhibited a great potential as multifunctional nanomaterials for multimodal molecular imaging and PPT 


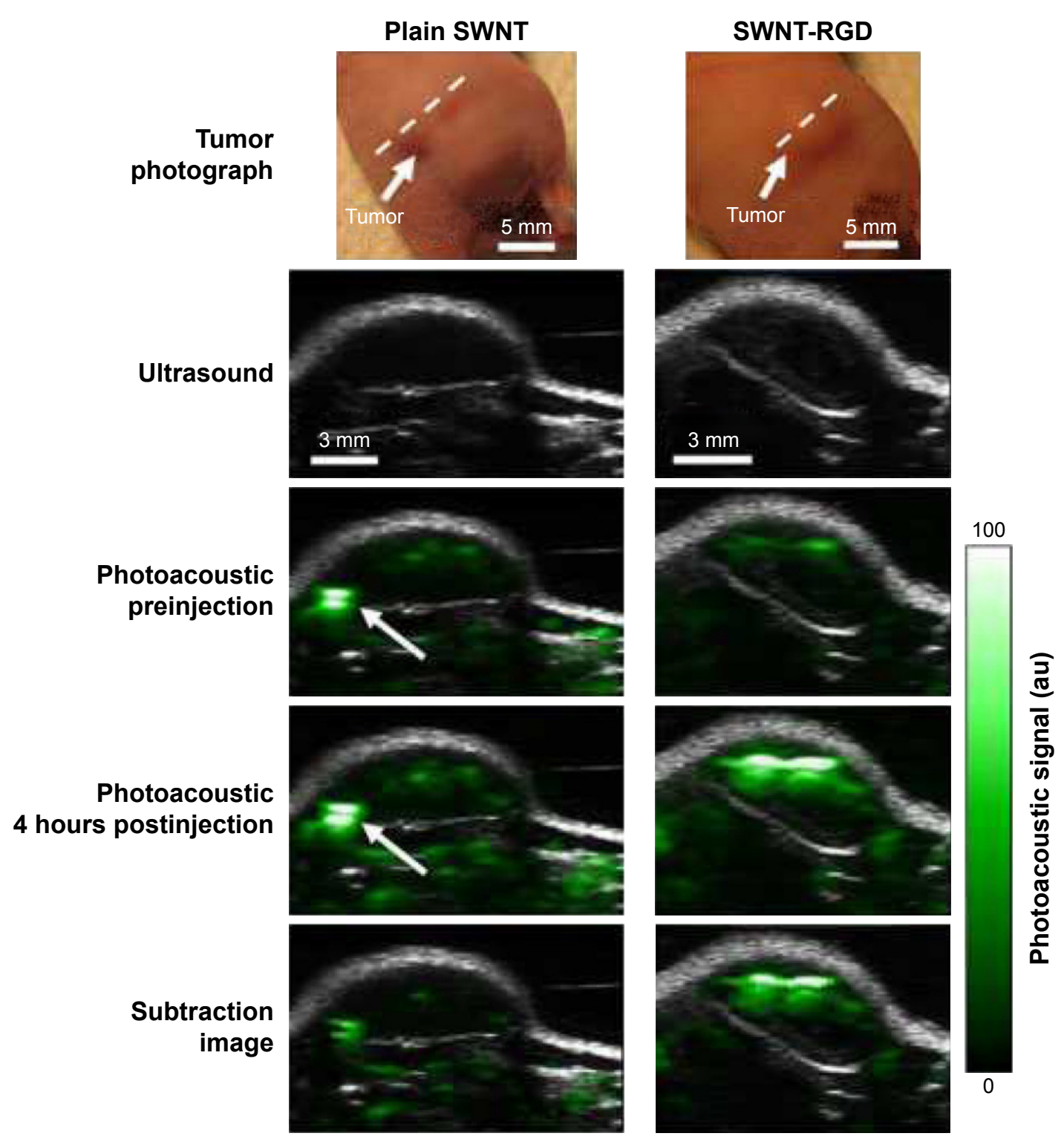

Figure 5 Images acquired before and after injecting SWNT-RGD as well as control plain SWNTs obtained by US (gray) and PA (green) imaging. Images with control plain SWNTs are shown on the left, while images with SWNT-RGD are shown on the right. Subtraction images were obtained to exclude background signals from the PA image. PA signals after injection of SWNT-RGD increase seven times higher than that of untargeted SWNTs. The PA signals in the mouse injected with plain SWNTs (pointed to by the white arrow) could not be seen in the subtraction image, indicating that the signals were not caused by SWNTs but by the blood vessels.

Notes: Reprinted with permission from Macmillan Publishers Ltd: Nat Nanotechnol. Zerda AD, Zavaleta C, Keren S, et al. Carbon nanotubes as photoacoustic molecular imaging agents in living mice. 3(9):557-562. Copyright 2008. ${ }^{44}$

Abbreviations: SWNT, single-walled carbon nanotube; RGD, arginine-glycine-aspartate; US, ultrasound; PA, photoacoustic.

because of their wide absorption bands and narrow emission bands as well as large surface areas. ${ }^{59}$

\section{Organic nanoparticles}

High excretion efficiency and prominent biocompatibility are characteristic properties of organic nanoparticles which determine their translation to clinical applications. Nevertheless, several issues such as low photostability, short circulation time and long-term retention in the tumor are still challenging for a successful application. Kim et al developed ICG-embedded organic nanoparticles via a photonic explorer for biomedical use by biologically localized embedding (PEBBLE) technique by encapsulating numerous dye molecules into a larger nanoparticle to significantly improve the photostability and circulation time. ${ }^{60}$

Perfluorocarbon (PFC) nanodroplets as promising probes for optical molecular imaging were integrated with plasmonic nanoparticles for US/PAI and therapy ${ }^{61,62} \mathrm{With}$ the growing translation to clinics, PFC nanodroplets have been refined with biocompatibility and nontoxicity ${ }^{63}$ It is noted that PFC nanodroplets display a unique profile of phase transition under ambient conditions, so it is possible to utilize them for 
size-controllable US and/or PAI and image-guided treatments by applying them to stimuli-responsive drug delivery to the diseased lesions.

\section{Inorganic magnetic nanoparticles}

Inorganic nanoparticles have been studied for years, whereas biosafety problems and poor drug-loading efficiency are obstacles to their actual clinical uses. ${ }^{64}$ As an exclusion, superparamagnetic iron oxide nanoparticles (SPIONs) have been approved by the US Food and Drug Administration and applied as nanoprobes for molecular PAI. ${ }^{65}$ SPIONs coated with silica were also employed in photoacoustic tomography (PAT), which showed great sensitivity with a minimum detectable concentration of $\sim 0.17$ and $\sim 0.23 \mathrm{mg} / \mathrm{mL}$ at depths of 5 and $10 \mathrm{~mm}$ inside the intralipids, respectively. ${ }^{66}$ The maximum penetration depth and optical stability of silicacoated SPIONs were studied as well.

Due to limited sample volume and inadequate numbers of CTCs, cancer diagnosis and metastasis prevention at an early stage are difficult. Hybrid AuNPs conjugated with magnetic nanoparticles have been developed to serve the multiplex targeting strategy and enable CTCs detection. By integrating both nanoparticles, urokinase plasminogen activator and folate receptors can be targeted without the blood cell noises, which provides high specificity in detecting CTCs. ${ }^{67}$

\section{Photosensitizers and activatable PA contrast agents}

The supramolecular self-assembled porphysome was synthesized by integrating porphyrin-lipid. ${ }^{68}$ Polyethylene glycol (PEG)-lipids were applied to improve the pharmacokinetic properties in imaging in vivo. Porphysome, with two separated layers of high-density material, exhibited two distinct optical absorption peaks. Moreover, the porphysomes had the ability of metal chelation, as was demonstrated by the shifts in optical density bands with metal ions inserted into the porphysome. Thermal energy, with similar efficacy as gold nanorods, was released by exposing porphysomes to laser irradiation when they were tested for their photochemical features. After optical stimulation, the amplitudes of porphysomes were almost six times higher than those of methylene blue, whereas the PA signal of porphysomes decreased six times after adding detergent with the signal of the methylene blue changing little. The experiment demonstrated the self-quenching feature of the porphysome to give off its PA signals.

\section{Graphene nanoparticles}

Graphene is a type of 2D carbon nanoparticle divided into three subclasses according to the diverse synthetic mode and morphology with corresponding distinct properties and responses on cells and tissues. ${ }^{69}$ Graphene nano-onions consist of concentric layers of graphene with a spherical shape. Graphene nanoplatelets (GONPs) are multiwalled graphene synthesized with graphite as the starting material in a disc shape. Graphene nanoribbons are composed of multilayered carbon nanotubes with graphene stacks like a ribbon. Different kinds of graphene nanoparticles have diverse morphologies, chemical states, particulate states, surface charges and numbers of layers which influence the cytotoxicity and cellular uptake. ${ }^{70-72}$ Graphene as a multifunctional agent has been applied to track stem cell as a contrast agent, evaluate the therapeutic effect and manipulate mesenchymal stem cells (MSCs) differentiation by delivering growth factors or genes into them. Despite their promising biomedical applications, graphene nanoparticles exhibit cytotoxicity dependent on their size and concentration instead of time. Fortunately, recent research reveals the possibility of employing the graphene nanoparticles for MSC-based imaging and therapy at potentially safe doses $(<50 \mathrm{mg} / \mathrm{mL})$ in vitro. ${ }^{73}$

The introduction of exogenous contrast agents expands the biomedical application realm and provides higher resolution in a deeper region of object tissue. Nevertheless, each exogenous contrast has its disadvantages. Different subclasses of AuNPs have diverse morphologies which enable their extensive applications, especially providing deeper penetration and lower scattering, but their optical properties are mainly based on complicated and costly coated layers, which is an obstacle to their medical application. SWNTs are inexpensive, but the problem of cytotoxicity is yet to be solved. ${ }^{73}$ Organic dyes are rather safe and have high excretion rate, allowing for their broad clinical applications. However, their small sizes $(<2 \mathrm{~nm})$ lead to their transportation into the echelon lymph nodes, which produces possible false positives. Moreover, their skin staining may cause irritation. Copper particles are innovative contrast agents synthesized in a highly stable way with a high commercial feasibility. Nevertheless, copper may cause neurotoxicity. ${ }^{74-76}$

\section{Biomedical applications of PAI in nanomedicine}

Since PAI has exhibited a series of excellent performances, pathological processes at molecular or cellular level can be detected with high-resolution PA images through incorporation of exogenous contrast agents, and various diseases may be diagnosed with the assistance of nanomaterials to serve nanomedicine. Studies have recently attached special attention to not only preclinical but also clinical 


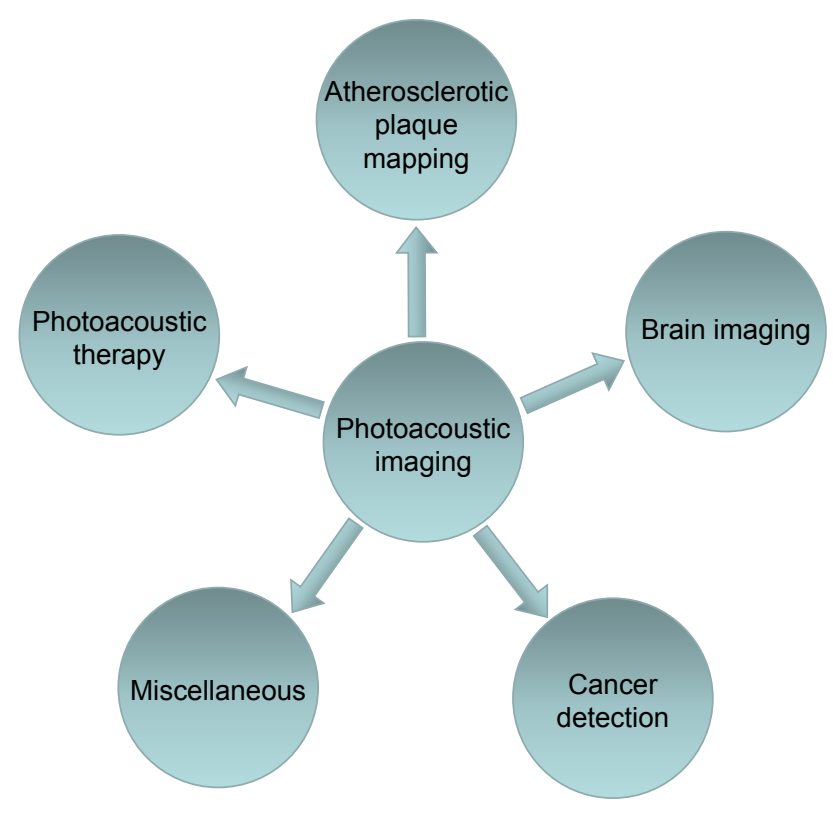

Figure 6 Domains of biomedical applications of photoacoustic imaging in nanomedicine.

applications in this regard, and breakthrough has been successfully achieved in main domains including cancer detection, atherosclerotic plaques mapping, brain imaging and PAI-based therapy (Figure 6). . $8,64,77^{5}$

\section{Cancer detection}

Cancer is an everlasting threat all over the world, and millions of people die of this vicious disease every year. ${ }^{78}$ For this reason, a successful and efficacious cancer detection strategy is desirable. Many modalities including magnetic resonance imaging, computed tomography, single-photon emission computed tomography and positron emission tomography have been used for patients; however, obvious deficiencies such as radiation risks, high expenses and insufficient precision limit the early-stage cancer detection. In contrast, PAI exhibits bright prospects in cancer monitoring, assessment of tumor margins and tumor metastasis.

The blood supply in neoplasm is quite active due to its neovascularization, and thus, endogenous PA contrast agents can be used to indicate the early development of superficial malignant tumors. Tripathi et al investigated melanoma detection by PAI using melanin as endogenous contrast agent, and verified the feasibility and accuracy by biopsy. ${ }^{79}$ Similarly, hemoglobin is a suitable indicator to demonstrate the degree of neovascularization in tumors, and the morphology and density increase of vasculature could be clearly displayed by means of PAI ${ }^{80}$ In addition, targeted exogenous contrast agents can be applied to further enhance the contrast so that tiny microvasculature in tumors can be visualized.
For example, through using RGD-labeled AuNPs, the integrin expression on the neovasculature of glioblastoma can be visualized by PA molecular imaging. ${ }^{81}$ Briefly, the specific targeting of RGD-labeled AuNPs to integrin causes plasmon resonance coupling between adjacent nanoparticles and changes their absorbance spectra which can be detected as a change in the PA signal amplitude. Zerda et al used RGDlabeled ICG-conjugated hybrid SWNTs for simultaneously targeting tumor vasculature and extravascular compartments of glioblastomas in living mice (Figure 7). ${ }^{82}$ In addition, the tumor progression and malignancy are of great significance for successful treatment and prognosis of patients, and hypoxia in the blood vessels prompts the progress and degree of malignancy. Hence, by monitoring the level of hemoglobin in tumor areas, PAI can be used to detect tumor progress. Siphanto et al acquired PAI information of tumor neovascularization in a rat through 10 days after inoculation with pancreatic tumor cells. ${ }^{83} \mathrm{Li}$ et al performed functional imaging of a mouse brain with a glioblastoma. The results clearly demonstrated that the tumor had a lower percentage of $\mathrm{SO}_{2}$ than the surrounding normal tissue. ${ }^{84}$

The intraoperational assessment of tumor margins is vitally important for determination of surgery range, recurrence of diseases and prognosis of patients. As a new technique, PAI displays great potential in the assessment of tumor margins especially for breast cancers. Due to the presence of fat, the blood supply in breast cancers is very rich which differs from normal breast tissues. As a consequence, a multispectral PAT has been performed by Li et al to assess breast tumor margin with the endogenous contrast agents of fat and hemoglobin. By discriminating PA signals of fat and hemoglobin in tumor and normal tissues, a distribution mapping of the whole breast was generated. Neoangiogenesis represented by the hemoglobin was clearly visualized from the fat composition in the normal tissues of breast, and the tumor margin was determined accurately which was identified by the histology. ${ }^{85}$

Tumor metastasis is another issue in clinical practice, and early-stage metastasis always occurs on the SLNs which is a tumor bed that receives lymphatic drainage from cancerous tissues. ${ }^{86,87}$ Since lymphatics do not possess endogenous photosensitive molecules, exogenous PA contrast agents may be introduced. Song et al performed an in vivo study using rats and successfully imaged SLNs with PAI system using the optically absorbing contrast agent methylene blue. ${ }^{88} \mathrm{Nam}$ et al showed that AuNPs endocytosed by MSCs could be distinguished as few as 200 cells within a tissue phantom. ${ }^{89}$ By targeting them to biomarkers overexpressed on the metastatic tumor cells, PA images could identify the cells 

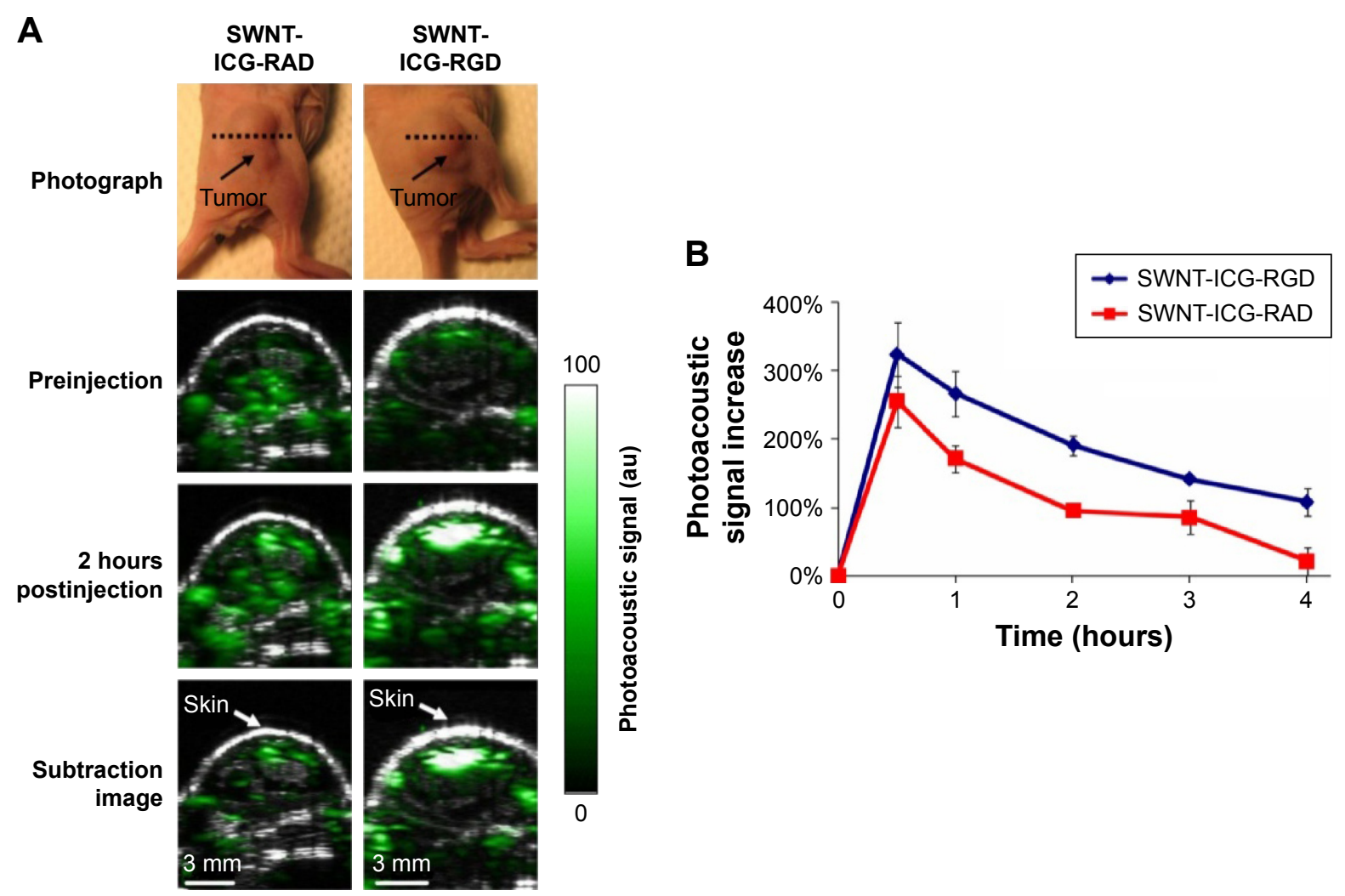

Figure 7 SWNT-ICG-RGD for targeting and imaging glioblastomas in living mice. (A) PA images of one vertical slice through the tumor (dotted black line) in mice injected with SWNT-ICG-RAD and SWNT-ICG-RGD. (B) PA signals of SWNT-ICG-RGD are significantly stronger than that of SWNT-ICG-RAD as a control (P<0.00I).

Notes: Reprinted with permission from de la Zerda A, Liu Z, Bodapati S, et al. Ultrahigh sensitivity carbon nanotube agents for photoacoustic molecular imaging in living mice. Nano Lett. 2010;10(6):2168-2172. Copyright 2010 American Chemical Society. ${ }^{82}$

Abbreviations: SWNT, single-walled carbon nanotube; ICG, indocyanine green; RGD, arginine-glycine-aspartate; PA, photoacoustic; RAD, arginine-alanine-aspartate.

metastasized to lymph nodes with high sensitivity and specificity (Figure 8). ${ }^{90}$ Furthermore, when used as an intraoperative imaging, PAI provided the information on whether a full lymphadenectomy was required immediately after the SLN identification by biopsy. ${ }^{22}$

\section{Atherosclerotic plaque mapping}

The carotid plaque rupture is extensively regarded as the main pathogenesis of acute cardiovascular events, and approximately $70 \%$ of the fatal acute myocardial infarction and sudden coronary death are caused by the plaque rupture. ${ }^{91}$ Nevertheless, recognition of atherosclerotic plaque at an early stage of development and the vulnerability assessment are still challenging nowadays. Intravascular photoacoustic endoscopic imaging can effectively display plaque microstructure, quantitatively analyze the relative/absolute contents of collagen, lipid and fiber and precisely identify vulnerable plaques. In particular, based on the characteristic optical absorptions of lipid in the NIR range, IVPAI is capable of imaging lipids in human coronary atherosclerosis ex vivo and is promisingly translated to in vivo clinics. ${ }^{92,93}$ Wang et al injected AuNPs on a diseased rabbit aorta to image macrophages which are important indicators of atherosclerotic formation at the early stage
(Figure 9). ${ }^{46}$ They showed that IVPAI validated the presence and the location of atherosclerotic plaques based on their outstanding contrast enhancement of AuNPs. Qin also prepared antibody-conjugated AuNPs for quantitative detection of MMP2 in atherosclerotic plaques in the abdominal aorta of a Watanabe heritable hyperlipidemic rabbit. ${ }^{94}$ Although IVPAI is promising for diagnosis of vulnerable plaques in the arterial wall by quantifying the lipid amounts and assessing their spatial distribution inside the arterial wall, the translation from bench to bedside has been limited due to slow imaging speed and lacking of a suitable laser source for lipid-specific first overtone excitation..$^{95}$

\section{Brain imaging and cerebral malfunction evaluation}

The ability of PAI to achieve high-resolution functional and metabolic imaging suggests it as a candidate for brain science studies. ${ }^{96,97}$ A real-time and clear visualization of brain vasculature is of great importance to study its functions and diagnose developing diseases. Lu et al performed PEGylated conjugation with hollow gold nanospheres (PEG-HAuNS) as the contrast agent to show the brain vasculature of nude mice. Five minutes after the injection of PEG-HAuNS, the PAT image displayed the 


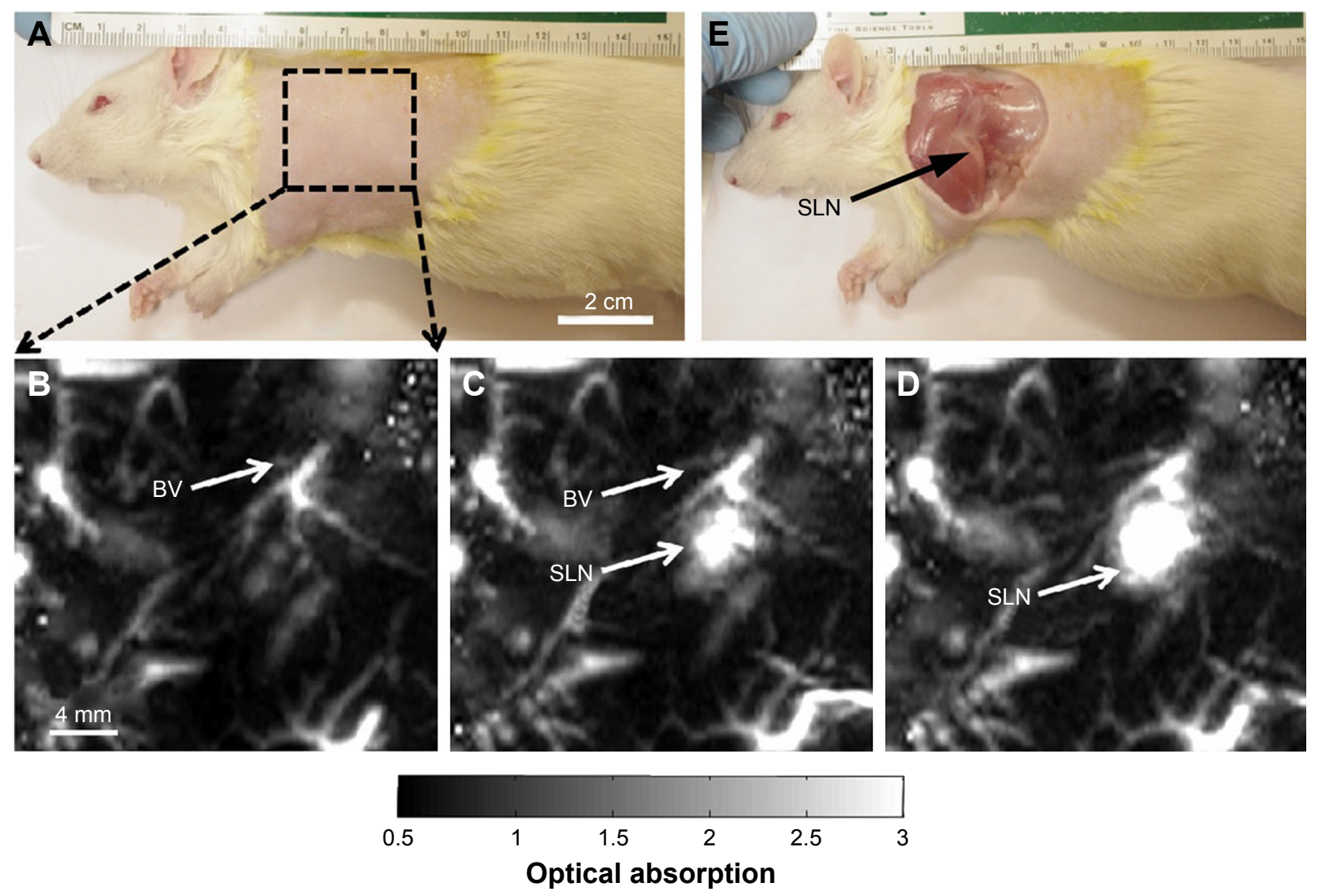

Figure 8 In vivo PAI of BV and SLN in rats with copper nanoparticles for tumor metastasis detection.

Notes: (A) Photograph of the rat with region of interest depilated before scanning. The scanning region is delineated by a black dotted square. (B) Baseline PA image acquired before NanoCuN injection. Bright region (arrow) represent blood vessel (BV). (C) PA image acquired almost immediately after NanoCuN administration. BV and SLN are marked with arrows. (D) PA image acquired 60 min after injection. The presence of SLN is more apparent in (D) than in (C). (E) Photograph of the rat with the skin excised after PA imaging. Reprinted with permission from Pan D, Cai X, Yalaz C, et al. Photoacoustic sentinel lymph node imaging with self-assembled copper neodecanoate nanoparticles. ACS Nano. 2012;6(2):1260-1267. Copyright 2012 American Chemical Society. ${ }^{90}$

Abbreviations: PAI, photoacoustic imaging; BV, blood vessel; SLN, sentinel lymph node.

A

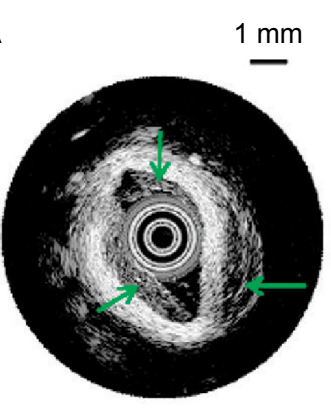

B

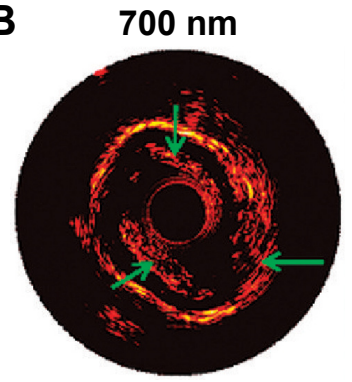

$\mathbf{E}$

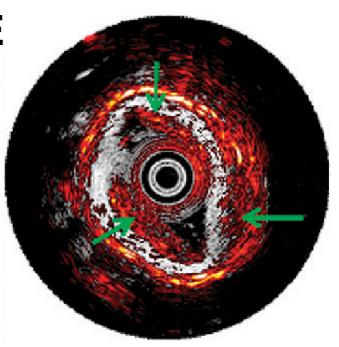

C

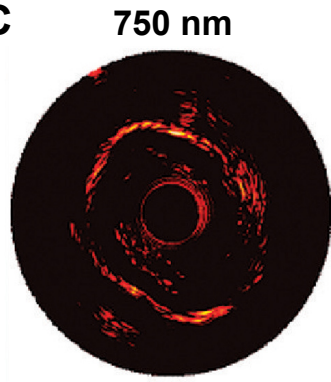

$\mathbf{F}$

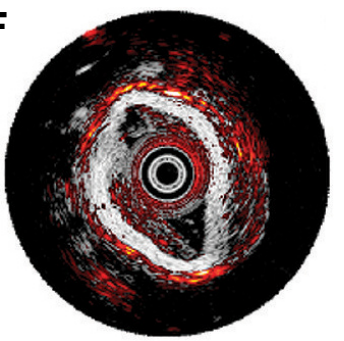

D

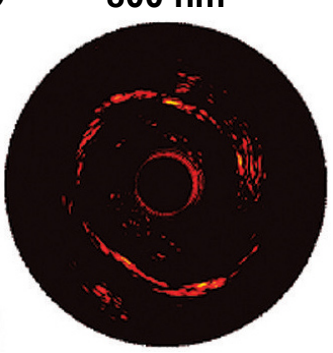

$\mathbf{G}$

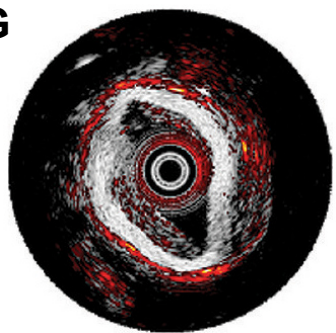

Figure 9 IVPAI of atherosclerotic plaques in a diseased rabbit aorta with AuNPs.

Notes: (A) The IVUS image of the rabbit aorta injected with macrophages loaded with Au NPs. (B-D) The normalized IVPA images of the aorta after injection. (E-G) The combined IVUS/IVPA images of the aorta after injection. The images (B-G) are obtained using 700, 750, and $800 \mathrm{~nm}$ wavelength, respectively. The injected macrophages in the outer and inner regions of aorta are denoted in the images (A, B, E) with green arrows. Reprinted with permission from Wang B, Yantsen E, Larson T, et al. Plasmonic intravascular photoacoustic imaging for detection of macrophages in atherosclerotic plaques. Nano Lett. 2009;9(6):2212-2217. Copyright 2009 American Chemical Society. ${ }^{46}$ Abbreviations: IVPAI, intravascular photoacoustic imaging; AuNPs, gold nanoparticles. 

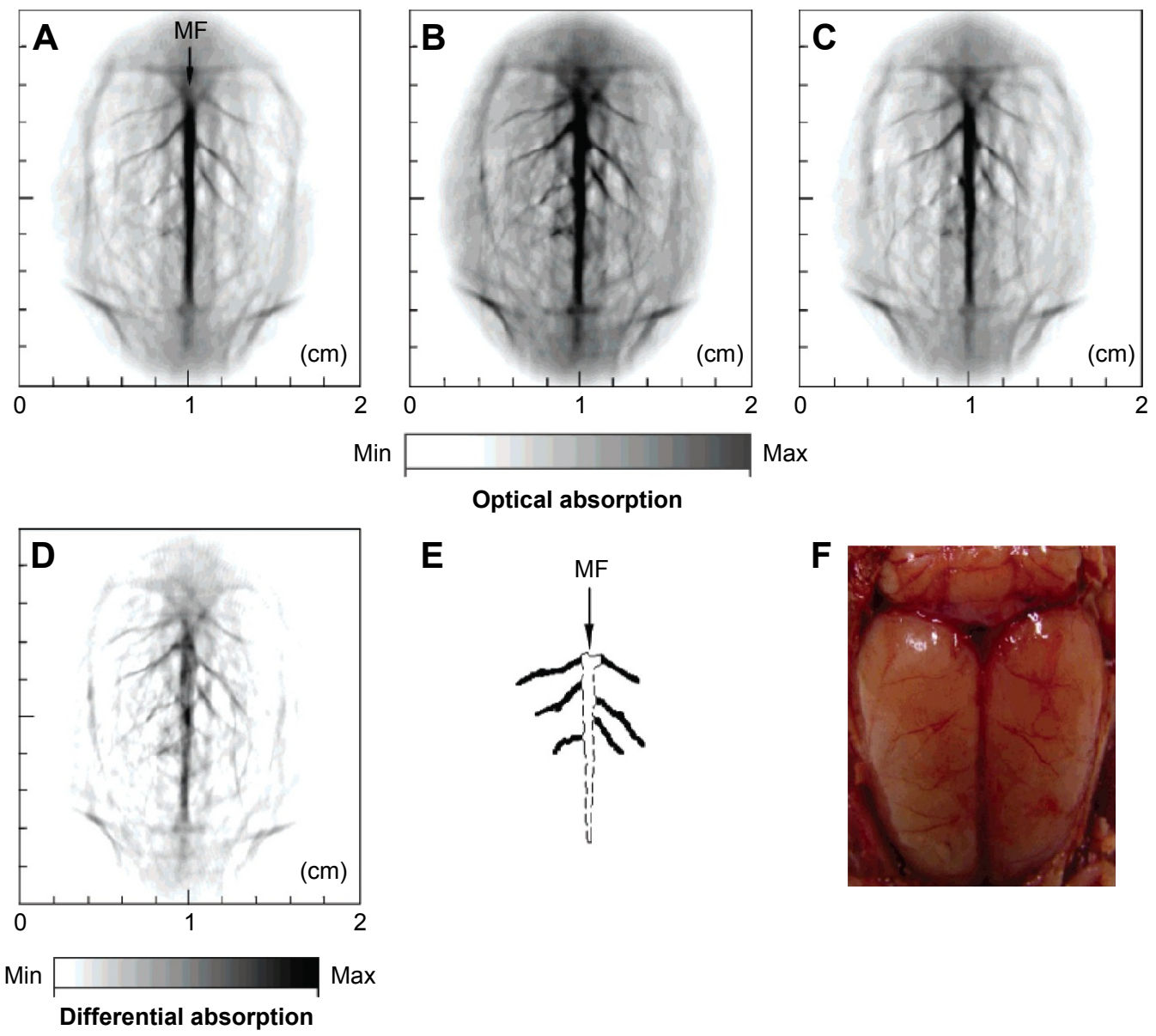

E

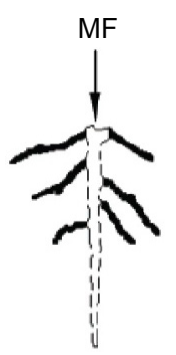

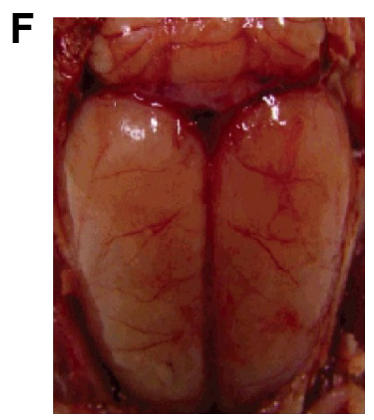

Figure 10 Noninvasive PAT imaging of a mouse brain in vivo employing the nanoshell contrast agent and NIR light at a wavelength of $800 \mathrm{~nm}$.

Notes: (A) Photoacoustic image acquired before the administrations of nanoshells. (B) Photoacoustic image obtained 20 min after injection. (C) Photoacoustic image acquired 350 min after injection. (D) Differential image obtained by subtracting the pre-injection image from the post-injection image. (Image $D=$ Image $B$ - Image $A$ ). (E) Large blood vessels in the cerebral cortex segmented from the PAT images (shown with solid regions). (F) Open-skull photograph of the rat brain cortex obtained after the data acquisition for PAT. Reprinted with permission from Wang Y, Xie X, Wang X, et al. Photoacoustic tomography of a nanoshell contrast agent in the in vivo rat brain. Nano Lett. 2004;4(9): 1689-1692. Copyright 2004 American Chemical Society. ${ }^{99}$

Abbreviations: PAT, photoacoustic tomography; NIR, near-infrared, MF, median fissure.

brain vasculature with great clarity. ${ }^{98}$ In addition to using hemoglobin as the endogenous contrast agent, exogenous AuNPs have been designed as PA contrast agents and even targeted drug carriers for brain drug transportation across blood-brain barrier, and thus, cerebral network mechanism and pathological natures can be revealed. Wang et al applied nanoshells to image the brain vasculatures of Sprague Dawley rats, and the full image of a mouse brain was emerged with the open-skull anatomical photograph and displayed functional information and processes in a rat brain system as shown in Figure 10.99

It is also valuable to evaluate brain activities by monitoring the change of functional biomarkers, such as total hemoglobin concentration $\left(\mathrm{Hb}_{\mathrm{T}}\right)$ and hemoglobin oxygen saturation $\left(\mathrm{SpO}_{2}\right)$. To assess the cerebral blood volume and $\mathrm{SpO}_{2}$ in brain, $\mathrm{PAI}$ can therefore be used to observe the brain activities. Studies have reported that intrinsic hemoglobin and extrinsic tumor-targeted nanoprobes were used for neuroimaging of Sprague Dawley rats at three different physiological states (hyperoxia, normoxia and hypoxia), the data of $\mathrm{Hb}_{\mathrm{T}}$ and $\mathrm{SpO}_{2}$ matched well and highly specific delineation of brain tumors was acquired, which suggested PAI as a powerful tool to help us understand the human neurological diseases and advance human neurological studies. ${ }^{100,101}$

Brain and spinal cord injuries causing paralysis or paraplegia are also a domain for PAI-aided evaluations. White matter loss is thought to be a critical event in spinal cord injury. ${ }^{102} \mathrm{Wu}$ et al used PAM at $1,730 \mathrm{~nm}$ excitation to assess white matter loss in spinal cord-injured rats. ${ }^{103}$ On the crosssectional images, contrast from white matter was $\sim 2.5$ times higher compared to grey matter, and the absorption difference was used to examine the morphology of white matter and changes in injured spinal cords.

\section{Miscellaneous}

Conventional endoscopy of gastrointestinal tract allows direct visualization of morphological changes and histological analysis 
of tissues, which is the gold standard for diagnosis. ${ }^{104}$ However, these methods are always influenced by technician's experience and lack of sensitivity. ${ }^{105}$ PAE may be useful as a minimal invasive diagnostic tool which provides higher spatial resolution than traditional US endoscopy. ${ }^{106}$ Yang et al successfully imaged the esophagi of New Zealand White rabbits and the descending colons of Sprague Dawley rats. The PAE provided anatomic information of rabbits' esophagus, surrounding tissue and proximal organs, and 3D images of the morphology and configuration of surrounded organs were obtained., ${ }^{3,107}$ Besides, early-stage gastrointestinal tumors and submucosal lesions were in situ characterized. The endoscopic imaging may be further improved with injection of exogenous targeted contrast agents so that malignant and premalignant lesions may be clearly detected.

Retinal diseases are the major causes of blindness. The existing retinal diagnostic methods such as fundus photography, scanning laser ophthalmoscopy and OCT are used for anatomical and functional ophthalmic imaging. There is an urgent demand for ophthalmic imaging of retinal tissues. ${ }^{108}$ Photoacoustic ophthalmoscopy (PAOM) offers a unique capability to measure optical absorption in the retina. Due to its compatibility with OCT and scanning laser ophthalmoscopy, co-registered multimodal images can be obtained from a single device with comprehensive retinal anatomic and functional information. ${ }^{109}$ Currently, researchers have realized in vivo retinal imaging by PAOM. For example, Jiao et al displayed the retinal vessels and retinal pigment epithelium in Long Evans rats' eyes by PAOM. ${ }^{108}$ Song et al combined PAOM with spectral domain OCT to simultaneously achieve the mapping of $\mathrm{SpO}_{2}$, retinal oxygen metabolic rate and the blood flow rate, which suggested this technique will serve as an integrated tool for visualizable diagnosis of retinal diseases. ${ }^{110}$

With the rapid development of PA instrument, highresolution PAI at depths of several millimeters has been realized. Researchers employed this high-resolution PAI for skin applications. iThera Medical has developed raster-scan optoacoustic mesoscopy (RSOM) imaging systems for highresolution visualization of the natural chromophores melanin and hemoglobin with up to $10 \mu \mathrm{m}$ resolution at depths of several millimeters, especially for detecting superficial microvasculature that is critical for diagnosis and treatment monitoring of diseases like skin cancers, tumor angiogenesis and vascular disease. Schwarz et al applied ultra-wideband RSOM to image healthy human skin at distinct locations and analyzed the anatomical information of different skin layers in vivo through different frequency ranges of the PA signals. By imaging a benign nevus, they found RSOM system was able to provide strong contrast of melanin-rich structures. Besides, they also identified RSOM can image the fine structures in the stratum corneum, dermal papillae and the horizontal plexus. ${ }^{111} \mathrm{Xu}$ et al recently developed a PA dermoscope equipped with an integrated PA probe and applied this PA dermoscope for imaging the skin in volunteers. The imaging results demonstrated the pigment distribution and thickness of the skin as well as vascular diameter and depth in the skin. Researchers believed that PA dermoscopy can serve as a potential tool for the diagnosis and curative effect evaluation of human skin diseases. ${ }^{112}$

Cell-based therapy offers a newly promising solution for treating diseases that failed to get cured at present. Cell-tracking technique can help in evaluating the effectiveness of treatments and providing essential knowledge of the basic principle and mechanism of action of cell therapy. Therefore, recent researches on cell tracking turn to be very hot. However, the development of an accurate and quantitative noninvasive cell-tracking technique is a highly challenging task. Currently, researchers are actively developing a variety of molecular contrast agents to be used for cell tracking with various forms of imaging modalities. Due to the properties of noninvasion and high resolution, PAI is also applied in researches of cell tracking. ${ }^{113}$ For instance, Comenge et al utilized gold nanorods as contrast agents for PA detection and monitoring of as few as $2 \times 10^{4}$ labeled MSCs in mice over 15 days. ${ }^{114} \mathrm{Pu}$ et al discussed the recent advances of SPNs as molecular imaging nano-agents in living animals and their applications ranging from SLN mapping to long-term cell tracking using PAI techniques. ${ }^{115}$

\section{PAl-based therapy}

Recently, the combination of diagnosis and therapy, that is, theranostics, has attracted much attention. As an efficient and commercial strategy, photoacoustics is also required to meet the "see-and-cure" concept. Such ideas have been realized with US targeted microbubble destruction, in which microbubbles were loaded with pharmaceutical payloads before US-triggered destruction and controlled release of drugs at the lesions. High-intensity focused US can also be used to synergistically generate sonoporation effect and enhance the massive drugs arrival at the diseased sites. For instance, AuNCs conjugated with anti-HER2 genes have been encapsulated into microbubbles for treatment of breast cancers. ${ }^{116}$ Cheng conducted an in vivo photothermal treatment using PEGylated WS2 nanosheets in a mouse tumor model and achieved excellent therapeutic efficacy with complete ablation of tumors. ${ }^{117}$

PPT as a moderate and facile treatment can also be incorporated with PAI. AuNPs and graphene oxides have been utilized for PAI and PAI-guided PTT of cancers. ${ }^{108}$ 
A

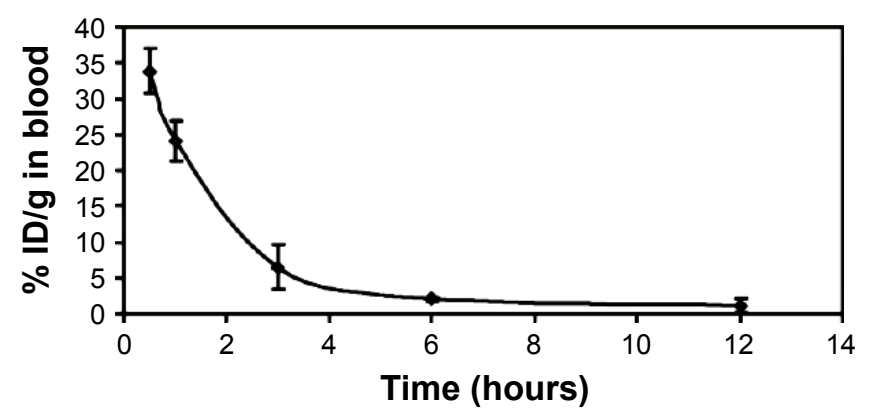

B 30 minutes

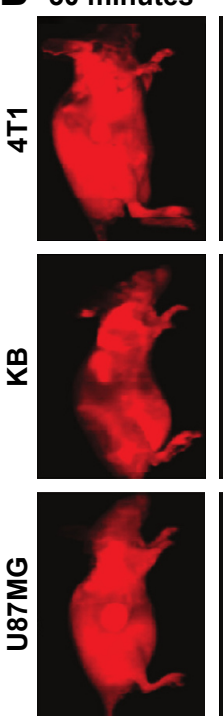

1 hour
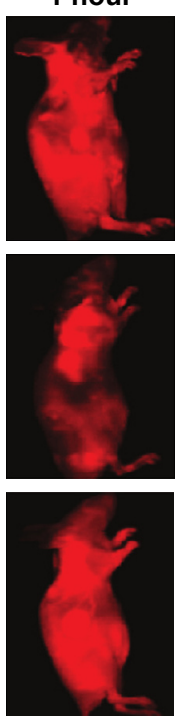

6 hours
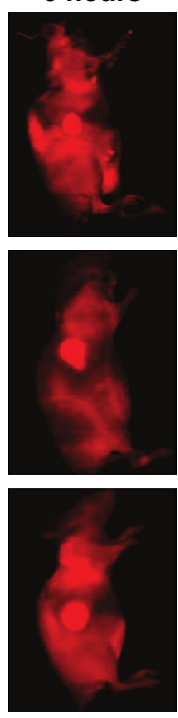

24 hours
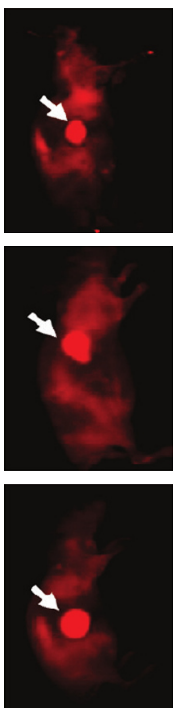

Figure I I In vivo PAI-based PPT of tumors in mice with NGS-PEG-Cy7.

Note: (A) The blood circulation curve of NGS-PEG-Cy7 determined by measuring Cy7 fluorescence in the blood at different time points post injection. (B) Spectrally unmixed fluorescence images of tumor (4TI, KB and U87MG) bearing BALB/C mice at different time points post injection of NGS-PEG-Cy7. Mouse autofluorescence was removed by spectral unmixing in the above images. High tumor uptake of NGSPEG-Cy7 was observed for all of the three tumor models. The arrows refer to the tumor region. Reprinted with permission from Yang K, Zhang S, Zhang G, Sun X, Lee ST, Liu Z. Graphene in mice: ultrahigh in vivo tumor uptake and efficient photothermal therapy. Nano Lett. 2010;10(9):3318-3323. Copyright 2010 American Chemical Society. ${ }^{118}$

Abbreviations: PAI, photoacoustic imaging; PPT, photothermal therapy; NGS, nanographene sheet; PEG, polyethylene glycol; Cy7, cyanine 7.

Yang et al firstly studied in vivo PAI-based therapy in several tumor-xenografted mice models using PEGylated nanographene sheets (NGSs). Based on the strong optical absorbance of NGSs in the NIR range, photothermal effect was strongly produced for tumor ablation (Figure 11). ${ }^{118}$

\section{Challenges and outlook}

In the past decade, photoacoustics has seen rapid developments in imaging and imaging-based therapy, and various nanomaterials have been utilized to serve the purposes in terms of nanomedicine. With acoustic deep penetration depth and optical high sensitivity, PAI can serve as a novel diagnostic strategy providing high-resolution and brilliant contrast images of lesions. Both anatomic and functional imaging can be realized, and moreover with the assistance of exogenous engineered nanomaterials, PAI can help in targeted imaging and therapy (ie, theranostics) for a broad spectrum of diseases. Harnessing all of these advantages, PAI has opened up a range of biomedical applications. In the cardiovascular disease, IVPAI may take the place of OCT and become a powerful tool for guiding percutaneous coronary intervention. In early detection of tumor, PAI of SLN can provide effective information on early metastasis. Besides, multispectral PAT opens a new way for clinical intraoperative breast tumor margin assessment. In addition, PA molecular imaging is able to characteristically target at the specific molecule of disease and can aid in early detection of critical diseases. It can be expected that PAI will witness extensive biomedical applications and actual translations to clinics in the coming years.

On the other hand, there are still some challenges for PAI to become a leading technology. Since the spatial resolution is determined by the nature of optical penetration depth, minute lesions (magnitude of millimeters) located in deep tissues can be hardly detected. In previous studies, the use of short-wavelength laser led to excellent signal-noise ratio (SNR), but the penetration depth was limited; in contrast, utilization of long-wavelength laser enhanced the penetration of laser in deep tissue, but the SNR was poor. Hence, the future research should focus on finding a laser with a suitable wavelength meeting the requirements of imaging depth and SNR at the same time. Another problem is how to further enhance the imaging sensitivity by developing cutting-edge PAI nanomaterials with excellent biosafety and appropriate half-life in vivo. When incorporated with therapeutic drugs, the capability of real-time monitoring and accurate evaluation of the fate of PAI nanomaterials in the body is still a bottleneck to be solved. In addition, further development in novel systems including laser sources, PA detectors and filter function as well as image reconstruction algorithms is an urgent problem demanding prompt solution. PAI systems need to become fast, stable and precise in real-time imaging, data acquisition and analysis, especially in quantitative data analysis. IVPAI and PAE are calling for miniaturized probes that are suitable for clinical application. 
In summary, bright perspectives and great opportunities along with challenges accompany the growing application of PAI, and it is believed that this technology will be playing a more active role in the transdisciplinary fields of nanomedicine, functional nanomaterials and advanced diagnostic instrumentations in their way toward human health care.

\section{Acknowledgments}

This study was supported by the National Natural Science Foundation of China (21575106); the Scientific Research Foundation for Returned Scholars, Ministry of Education of China, Wenzhou City Science \& Technology Bureau, China (No Y20150084, No Y20150085); and Health and Family Planning Commission of Zhejiang Province, China (No 2015KYA159, No 2016KYA143).

\section{Disclosure}

The authors report no conflicts of interest in this work.

\section{References}

1. Wang LV, Hu S. Photoacoustic tomography: in vivo imaging from organelles to organs. Science. 2012;335(6075):1458-1462.

2. Wilson KE, Wang TY, Willmann JK. Acoustic and photoacoustic molecular imaging of cancer. J Nucl Med. 2013;54(11):1851-1854.

3. Yang JM, Favazza C, Chen R, et al. Simultaneous functional photoacoustic and ultrasonic endoscopy of internal organs in vivo. Nat Med 2012;18(8):1297-1302.

4. Wang LV. Prospects of photoacoustic tomography. Med Phys. 2008; 35(12):5758-5767.

5. Ku G, Maslov K, Li L, Wang LV. Photoacoustic microscopy with 2- $\mu \mathrm{m}$ transverse resolution. J Biomed Opt. 2010;15(2):021302.

6. Esenaliev RO, Larina IV, Larin KV, Deyo DJ, Motamedi M, Prough DS. Optoacoustic technique for noninvasive monitoring of blood oxygenation: a feasibility study. Appl Opt. 2002;41(22):4722-4731.

7. Zhang HF, Wang J, Wei Q, Liu T, Jiao S, Puliafito CA. Collecting back-reflected photons in photoacoustic microscopy. Opt Express. 2010 18(2):1278-1282.

8. Sun Y, Sobel ES, Jiang H. First assessment of three-dimensional quantitative photoacoustic tomography for in vivo detection of osteoarthritis in the finger joints. Med Phys. 2011;38(7):4009-4017.

9. Van de Sompel D, Sasportas LS, Dragulescu-Andrasi A, Bohndiek S, Gambhir SS. Improving image quality by accounting for changes in water temperature during a photoacoustic tomography scan. PLoS One. 2012;7(10):e45337.

10. Neuschmelting V, Burton NC, Lockau H, et al. Performance of a multispectral optoacoustic tomography (MSOT) system equipped with 2D vs 3D handheld probes for potential clinical translation. Photoacoustics. 2016;4(1):1-10.

11. Singh MKA, Steenbergen W, Manohar S. Handheld probe-based dual mode ultrasound/photoacoustics for biomedical imaging. In: Olivo M, Dinish US, editors. Frontiers in Biophotonics for Translational Medicine. Singapore: Springer; 2015:209-247.

12. Mallidi S, Watanabe K, Timerman D, Schoenfeld D, Hasan T. Prediction of tumor recurrence and therapy monitoring using ultrasound-guided photoacoustic imaging. Theranostics. 2015;5(3):289-301.

13. Paproski RJ, Heinmiller A, Wachowicz K, Zemp RJ. Multi-wavelength photoacoustic imaging of inducible tyrosinase reporter gene expression in xenograft tumors. Sci Rep. 2014;4:5329.

14. Kimura $Y$, Kurimoto $T$, Imai $Y$, et al. Novel biocompatible cobalt oxide nanoparticles for use in dual photoacoustic and magnetic resonance imaging. JSM Biotechnol Bioeng. 2014;2(2):1043-1047.
15. McNally LR, Mezera M, Morgan DE, et al. Current and emerging clinical applications of multispectral optoacoustic tomography (MSOT) in oncology. Clin Cancer Res. 2016;22(14):3432-3439.

16. Ermilov SA, Su R, Conjusteau A, et al. Three-dimensional optoacoustic and laser-induced ultrasound tomography system for preclinical research in mice: design and phantom validation. Ultrason Imaging. 2016;38(1): $77-95$.

17. Luke GP, Yeager D, Emelianov SY. Biomedical applications of photoacoustic imaging with exogenous contrast agents. Ann Biomed Eng 2012;40(2):422-437.

18. Rao B, Soto F, Kerschensteiner D, Wang LV. Integrated photoacoustic, confocal, and two-photon microscope. J Biomed Opt. 2014; 19(3):36002.

19. Cai X, Zhang Y, Li L, et al. Investigation of neovascularization in threedimensional porous scaffolds in vivo by a combination of multiscale photoacoustic microscopy and optical coherence tomography. Tissue Eng Part C Methods. 2013;19(3):196-204.

20. Wang Y, Maslov K, Kim C, Hu S, Wang LV. Integrated photoacoustic and fluorescence confocal microscopy. IEEE Trans Biomed Eng. 2010;57(10):2576-2578.

21. Jeon M, Song W, Huynh E, et al. Methylene blue microbubbles as a model dual-modality contrast agent for ultrasound and activatable photoacoustic imaging. J Biomed Opt. 2014;19(1):16005.

22. Bayer CL, Joshi PP, Emelianov SY. Photoacoustic imaging: a potential tool to detect early indicators of metastasis. Expert Rev Med Devices. 2013;10(1):125-134.

23. Langer G, Buchegger B, Jacak J, Klar TA, Berer T. Frequency domain photoacoustic and fluorescence microscopy. Biomed Opt Express. 2016; 7(7):2692-2702.

24. Hudson SV, Huang JS, Yin W, et al. Targeted noninvasive imaging of EGFR-expressing orthotopic pancreatic cancer using multispectral optoacoustic tomography. Cancer Res. 2014;74(21):6271-6279.

25. Akers WJ, Kim C, Berezin M, et al. Noninvasive photoacoustic and fluorescence sentinel lymph node identification using dye-loaded perfluorocarbon nanoparticles. ACS Nano. 2011;5(1):173-182.

26. Gamelin J, Maurudis A, Aguirre A, et al. A real-time photoacoustic tomography system for small animals. Opt Express. 2009;17(13): 10489-10498.

27. Taruttis A, Herzog E, Razansky D, Ntziachristos V. Real-time imaging of cardiovascular dynamics and circulating gold nanorods with multispectral optoacoustic tomography. Opt Express. 2010;18(19): 19592-19602.

28. Li R, Phillips E, Wang P, Goergen CJ, Cheng JX. Label-free in vivo imaging of peripheral nerve by multispectral photoacoustic tomography. J Biophotonics. 2016;9(1-2):124-128.

29. Hu S, Wang LV. Photoacoustic imaging and characterization of the microvasculature. J Biomed Opt. 2010;15(1):011101.

30. Maslov K, Zhang HF, Hu S, Wang LV. Optical-resolution photoacoustic microscopy for in vivo imaging of single capillaries. Opt Lett. 2008;33(9):929-931.

31. Zhang C, Maslov K, Wang LV. Subwavelength-resolution label-free photoacoustic microscopy of optical absorption in vivo. Opt Lett. 2010; 35(19):3195-3197.

32. Zhang HF, Maslov K, Stoica G, Wang LV. Functional photoacoustic microscopy for high-resolution and noninvasive in vivo imaging. Nat Biotechnol. 2006;24(7):848-851.

33. Li L, Zemp RJ, Lungu G, Stoica G, Wang LV. Photoacoustic imaging of lac Z gene expression in vivo. J Biomed Opt. 2007;12(2):020504.

34. Li M, Oh J, Xie X, et al. Simultaneous molecular and hypoxia imaging of brain tumors in vivo using spectroscopic photoacoustic tomography. Proc IEEE. 2008;96(3):481-489.

35. Homan K, Gomez S, Gensler H, Shah J, Brannon-Peppas L, Emelianov S. Design and development of multifunctional contrast agents for photoacoustic imaging. Proc SPIE. 2009;7190:71900I.

36. Schultz CJ, Serruys PW, van der Ent M, et al. First-in-man clinical use of combined near-infrared spectroscopy and intravascular ultrasound: a potential key to predict distal embolization and no-reflow? J Am Coll Cardiol. 2010;56(4):314. 
37. Mehrmohammadi M, Yoon KY, Qu M, Johnston KP, Emelianov SY. Enhanced pulsed magnetomotive ultrasound imaging using superparamagnetic nanoclusters. Nanotechnology. 2011;22(4):045502.

38. Park S, Kim J, Jeon M, Song J, Kim C. In vivo photoacoustic and fluorescence cystography using clinically relevant dual modal indocyanine green. Sensors (Basel). 2014;14(10):19660-19668.

39. Song W, Wei Q, Liu $T$, et al. Integrating photoacoustic ophthalmoscopy with scanning laser ophthalmoscopy, optical coherence tomography, and fluorescein angiography for a multimodal retinal imaging platform. J Biomed Opt. 2012;17(6):061206.

40. Ku G, Fornage BD, Jin X, Xu M, Hunt KK, Wang LV. Thermoacoustic and photoacoustic tomography of thick biological tissues toward breast imaging. Technol Cancer Res Treat. 2005;4(5):559-566.

41. Lungu GF, Li ML, Xie X, Wang LV, Stoica G. In vivo imaging and characterization of hypoxia-induced neovascularization and tumor invasion. Int J Oncol. 2007;30(1):45-54.

42. Schneider BP, Miller KD. Angiogenesis of breast cancer. J Clin Oncol. 2005;23(8): 1782-1790.

43. Agarwal A, Huang SW, O'Donnell M, et al. Targeted gold nano-rod contrast agent for prostate cancer detection by photoacoustic imaging. J Appl Phys. 2007;102(6):064701-064704.

44. Zerda AD, Zavaleta $\mathrm{C}$, Keren $\mathrm{S}$, et al. Carbon nanotubes as photoacoustic molecular imaging agents in living mice. Nat Nanotechnol. 2008;3(9): $557-562$.

45. Rostro-Kohanloo BC, Bickford LR, Payne CM, et al. The stabilization and targeting of surfactant-synthesized gold nanorods. Nanotechnology. 2009;20(43):434005.

46. Wang B, Yantsen E, Larson T, et al. Plasmonic intravascular photoacoustic imaging for detection of macrophages in atherosclerotic plaques. Nano Lett. 2009;9(6):2212-2217.

47. Yeager D, Karpiouk A, Wang B, et al. Intravascular photoacoustic imaging of exogenously labeled atherosclerotic plaque through luminal blood. J Biomed Opt. 2012;17(10):106016.

48. Pan D, Pramanik M, Senpan A, et al. Molecular photo acoustic imaging (PAI) with ligand-directed gold nanobeacons. Angew Chem Int Ed. 2009;48:4170-4173.

49. Skrabalak SE, Chen J, Sun Y, et al. Gold nanocages: synthesis, properties, and applications. Acc Chem Res. 2008;41(12):1587-1595.

50. Chen J, Yang M, Zhang Q, et al. Gold nanocages: a novel class of multifunctional nanomaterials for theranostic applications. Adv Funct Mater. 2010;20:3684-3694.

51. Song KH, Kim C, Cobley CM, Xia Y, Wang LV. Near-infrared gold nanocages as a new class of tracers for photoacoustic sentinel lymph node mapping on a rat model. Nano Lett. 2009;9(1):183-188.

52. Li ML, Wang JC, Schwartz JA, et al. In-vivo photoacoustic microscopy of nanoshell extravasation from solid tumor vasculature. J Biomed Opt. 2009;14(1):010507.

53. O'Neal DP, Hirsch LR, Halas NJ, Payne JD, West JL. Photo-thermal tumor ablation in mice using near infrared-absorbing nanoparticles. Cancer Lett. 2004;209(2):171-176.

54. Pan D, Pramanik M, Senpan A, et al. Molecular photoacoustic imaging of angiogenesis with integrin targeted gold nanobeacons. FASEB J. 2011;25(3):875-882.

55. Kim JW, Galanzha EI, Shashkov EV, Moon HM, Zharov VP. Golden carbon nanotubes as multimodal photoacoustic and photothermal high-contrast molecular agents. Nat Nanotechnol. 2009;4(10):688-694.

56. Liu Z, Sun X, Nakayama-Ratchford N, Dai H. Supramolecular chemistry on water-soluble carbon nanotubes for drug loading and delivery. ACS Nano. 2007;1(1):50-56.

57. Yu J, Wu C, Zhang X, et al. Stable functionalization of small semiconducting polymer dots via covalent cross-linking and their application for specific cellular imaging. Adv Mater. 2012;24(26): 3498-3504

58. Mallidi S, Luke GP, Emelianov S. Photoacoustic imaging in cancer detection, diagnosis, and treatment guidance. Trends Biotechnol. 2011;29(5): $213-221$.
59. Onoshima D, Yukawa H, Baba Y. Multifunctional quantum dots-based cancer diagnostics and stem cell therapeutics for regenerative medicine. Adv Drug Deliv Rev. 2015;95(1):2-14.

60. Kim G, Huang SW, Day KC, et al. Indocyanine-green-embedded PEBBLEs as a contrast agent for photoacoustic imaging. $J$ Biomed Opt. 2007;12(4):044020.

61. Wilson K, Homan K, Emelianov S. Biomedical photoacoustics beyond thermal expansion using triggered nanodroplet vaporization for contrastenhanced imaging. Nat Commun. 2012;3:618.

62. Zhong J, Yang S, Zheng X, Zhou T, Xing D. In vivo photoacoustic therapy with cancer-targeted indocyanine green containing nanoparticles. Nanomedicine (Lond). 2013;8(6):903-919.

63. Hannah A, Luke G, Wilson K, Homan K, Emelianov S. Indocyanine green-loaded photoacoustic nanodroplets: dual contrast nanoconstructs for enhanced photoacoustic and ultrasound imaging. ACS Nano. 2014; 8(1):250-259.

64. Wang B, Karpiouk A, Yeager D, et al. Intravascular photoacoustic imaging of lipid in atherosclerotic plaques in the presence of luminal blood. Opt Lett. 2012;37(7):1244-1246.

65. Alwi R, Telenkov S, Mandelis A, et al. Silica-coated super paramagnetic iron oxide nanoparticles (SPION) as biocompatible contrast agent in biomedical photoacoustics. Biomed Opt Express. 2012;3(10):2500-2509.

66. Feng L, Zhu C, Yuan H, Liu L, Lv F, Wang S. Conjugated polymer nanoparticles: preparation, properties, functionalization and biological applications. Chem Soc Rev. 2013;42(16):6620-6633.

67. Galanzha EI, Shashkov EV, Kelly T, Kim JW, Yang L, Zharov VP. In vivo magnetic enrichment and multiplex photoacoustic detection of circulating tumour cells. Nat Nanotechnol. 2009;4(12): $855-860$.

68. Lovell JF, Jin CS, Huynh E, et al. Porphysome nanovesicles generated by porphyrin bilayers for use as multimodal biophotonic contrast agents. Nat Mater. 2011;10(4):324-332.

69. Wang X, Podila R, Shannahan JH, Rao AM, Brown JM. Intravenously delivered graphene nanosheets and multiwalled carbon nanotubes induce site-specific Th2 inflammatory responses via the IL-33/ST2 axis. Int J Nanomedicine. 2013;8:1733-1748.

70. Mullick Chowdhury S, Lalwani G, Zhang K, Yang JY, Neville K, Sitharaman B. Cell specific cytotoxicity and uptake of graphene nanoribbons. Biomaterials. 2013;34(1):283-293.

71. Mao HY, Laurent S, Chen W, et al. Graphene: promises, facts, opportunities, and challenges in nanomedicine. Chem Rev. 2013;113(5): 3407-3424.

72. Talukdar Y, Rashkow J, Lalwani G, Kanakia S, Sitharaman B. The effects of graphene nanostructures on mesenchymal stem cells. Biomaterials. 2014;35(18):4863-4877.

73. Yang ST, Luo J, Zhou Q, Wang H. Pharmacokinetics, metabolism and toxicity of carbon nanotubes for biomedical purposes. Theranostics. 2012;2(3):271-282.

74. Lalwani G, Cai X, Nie L, Wang LV, Sitharaman B. Graphene-based contrast agents for photoacoustic and thermoacoustic tomography. Photoacoustics. 2013;1(3-4):62-67.

75. Lalwani G, D'Agati M, Khan AM, et al. Toxicology of graphene-based nanomaterials. Adv Drug Deliv Rev. 2016;105(Pt B):109-144.

76. Wang YW, Fu YY, Peng QL, et al. Dye-enhanced graphene oxide for photothermal therapy and photoacoustic imaging. $J$ Mater Chem $B$. 2013;1(42):5762-5767.

77. Li C, Wang LV. Photoacoustic tomography and sensing in biomedicine. Phys Med Biol. 2009;54(19):R59-R97.

78. Hanahan D, Weinberg RA. The hallmarks of cancer. Cell. 2000; 100(1):57-70

79. Tripathi SV, Zhou Y, Linette GP, et al. Handheld dual-modality ultrasound and photoacoustic imaging for in vivo determination of melanoma depth. J Invest Dermatol. 2016;136(5):S107.

80. Lao Y, Xing D, Yang S, Xiang L. Noninvasive photoacoustic imaging of the developing vasculature during early tumor growth. Phys Med Biol. 2008;53(15):4203-4212. 
81. Beard P. Biomedical photoacoustic imaging. Interface Focus. 2011; 1(4):602-631.

82. de la Zerda A, Liu Z, Bodapati S, et al. Ultrahigh sensitivity carbon nanotube agents for photoacoustic molecular imaging in living mice. Nano Lett. 2010;10(6):2168-2172.

83. Siphanto RI, Thumma KK, Kolkman RG, et al. Serial noninvasive photoacoustic imaging of neovascularization in tumor angiogenesis. Opt Express. 2005;13(1):89-95.

84. Li ML, Oh JT, Xie X, et al. Simultaneous molecular and hypoxia imaging of brain tumors in vivo using spectroscopic photoacoustic tomography. Proc IEEE. 2008;96(3):481-489.

85. Li R, Wang P, Lan L, et al. Assessing breast tumor margin by multispectral photoacoustic tomography. Biomed Opt Express. 2015;6(4): 1273-1281.

86. Wittekind C, Neid M. Cancer invasion and metastasis. Oncology. 2005;69(Suppl 1):14-16.

87. Veronesi U, Paganelli G, Galimberti V, et al. Sentinel-node biopsy to avoid axillary dissection in breast cancer with clinically negative lymph-nodes. Lancet. 1997;349(9069):1864-1867.

88. Song KH, Stein EW, Margenthaler JA, Wang LV. Noninvasive photoacoustic identification of sentinel lymph nodes containing methylene blue in vivo in a rat model. J Biomed Opt. 2008;13(5):054033.

89. Nam SY, Ricles LM, Suggs LJ, Emelianov SY. In vivo ultrasound and photoacoustic monitoring of mesenchymal stem cells labeled with gold nanotracers. PLoS One. 2012;7(5):e37267.

90. Pan D, Cai X, Yalaz C, et al. Photoacoustic sentinel lymph node imaging with self-assembled copper neodecanoate nanoparticles. ACS Nano. 2012;6(2):1260-1267.

91. Aikawa M, Libby P. The vulnerable atherosclerotic plaque: pathogenesis and therapeutic approach. Cardiovasc Pathol. 2004;13(3): $125-138$

92. Jansen K, van der Steen AF, van Beusekom HM, Oosterhuis JW, van Soest G. Intravascular photoacoustic imaging of human coronary atherosclerosis. Opt Lett. 2011;36(5):597-599.

93. Wu M, Jansen K, van der Steen AF, van Soest G. Specific imaging of atherosclerotic plaque lipids with two-wavelength intravascular photoacoustics. Biomed Opt Express. 2015;6(9):3276-3286.

94. Qin H, Zhao Y, Zhang J, Pan X, Yang S, Xing D. Inflammation-targeted gold nanorods for intravascular photoacoustic imaging detection of matrix metalloproteinase-2 (MMP 2) in atherosclerotic plaques. Nanomedicine. 2016;12(7):1765-1774.

95. Hui J, Li R, Phillips EH, Goergen CJ, Sturek M, Cheng JX. Bondselective photoacoustic imaging by converting molecular vibration into acoustic waves. Photoacoustics. 2016;4(1):11-21.

96. Yao J, Wang LV. Photoacoustic brain imaging: from microscopic to macroscopic scales. Neurophotonics. 2014;1(1):011003.

97. Wang LV, Gao L. Photoacoustic microscopy and computed tomography: from bench to bedside. Annu Rev Biomed Eng. 2014;16: $155-185$.

98. Lu W, Huang Q, Ku G, et al. Photoacoustic imaging of living mouse brain vasculature using hollow gold nanospheres. Biomaterials. 2010; 31(9):2617-2626.

99. Wang Y, Xie X, Wang X, et al. Photoacoustic tomography of a nanoshell contrast agent in the in vivo rat brain. Nano Lett. 2004;4(9): 1689-1692.
100. Kim C, Favazza C, Wang LV. In vivo photoacoustic tomography of chemicals: high-resolution functional and molecular optical imaging at new depths. Chem Rev. 2010;110(5):2756-2782.

101. Wang D, Wu Y, Xia J. Review on photoacoustic imaging of the brain using nanoprobes. Neurophotonics. 2016;3(1):010901.

102. Liu NK, Zhang YP, Titsworth WL, et al. A novel role of phospholipase A2 in mediating spinal cord secondary injury. Ann Neurol. 2006;59(4):606-619.

103. Wu W, Wang P, Cheng JX, Xu XM. Assessment of white matter loss using bond-selective photoacoustic imaging in a rat model of contusive spinal cord injury. J Neurotrauma. 2014;31(24):1998-2002.

104. Valdastri P, Simi M, Webster RJ 3rd. Advanced technologies for gastrointestinal endoscopy. Annu Rev Biomed Eng. 2012;14(1):397-429.

105. Yoon TJ, Cho YS. Recent advances in photoacoustic endoscopy. World J Gastrointest Endosc. 2013;5(11):534-539.

106. Yang JM, Maslov K, Yang HC, Zhou Q, Shung KK, Wang LV. Photoacoustic endoscopy. Opt Lett. 2009;34(10):1591-1593.

107. Yang JM, Chen R, Favazza C, et al. A 2.5-mm diameter probe for photoacoustic and ultrasonic endoscopy. Opt Express. 2012;20(21): 23944-23953.

108. Jiao S, Jiang M, Hu J, et al. Photoacoustic ophthalmoscopy for in vivo retinal imaging. Opt Express. 2010;18(4):3967-3972.

109. Zhang HF, Puliafito CA, Jiao S. Photoacoustic ophthalmoscopy for in vivo retinal imaging: current status and prospects. Ophthalmic Surg Lasers Imaging. 2011;42(Suppl 4):S106-S115.

110. Song W, Wei Q, Liu W, et al. A combined method to quantify the retinal metabolic rate of oxygen using photoacoustic ophthalmoscopy and optical coherence tomography. Sci Rep. 2014;4(40):6525.

111. Schwarz M, Omar M, Buehler A, Aguirre J, Ntziachristos V. Implications of ultrasound frequency in optoacoustic mesoscopy of the skin. IEEE Trans Med Imaging. 2015;34(2):672-677.

112. Xu D, Yang S, Wang Y, Gu Y, Xing D. Noninvasive and high-resolving photoacoustic dermoscopy of human skin. Biomed Opt Express. 2016;7(6):2095-2102.

113. Meir R, Motiei M, Popovtzer R. Gold nanoparticles for in vivo cell tracking. Nanomedicine (Lond). 2014;9(13):2059-2069.

114. Comenge J, Fragueiro O, Sharkey J, et al. Preventing plasmon coupling between gold nanorods improves the sensitivity of photoacoustic detection of labeled stem cells in vivo. ACS Nano. 2016;10(7):7106-7116.

115. Pu K, Chattopadhyay N, Rao J. Recent advances of semiconducting polymer nanoparticles in in vivo molecular imaging. J Control Release. 2016;240:312-322.

116. Wu D, Huang L, Jiang MS, Jiang H. Contrast agents for photoacoustic and thermoacoustic imaging: a review. Int J Mol Sci. 2014; 15(12):23616-23639.

117. Cheng L, Liu J, Gu X, et al. PEGylated WS2 nanosheets as a multifunctional theranostic agent for in vivo dual-modal CT/ photoacoustic imaging guided photothermal therapy. Adv Mater. 2014; 26(12):1886-1893.

118. Yang K, Zhang S, Zhang G, Sun X, Lee ST, Liu Z. Graphene in mice: ultrahigh in vivo tumor uptake and efficient photothermal therapy. Nano Lett. 2010;10(9):3318-3323.
International Journal of Nanomedicine

\section{Publish your work in this journal}

The International Journal of Nanomedicine is an international, peerreviewed journal focusing on the application of nanotechnology in diagnostics, therapeutics, and drug delivery systems throughout the biomedical field. This journal is indexed on PubMed Central, MedLine, CAS, SciSearch $₫$, Current Contents $₫ /$ Clinical Medicine,

\section{Dovepress}

Journal Citation Reports/Science Edition, EMBase, Scopus and the Elsevier Bibliographic databases. The manuscript management system is completely online and includes a very quick and fair peer-review system, which is all easy to use. Visit http://www.dovepress.com/ testimonials.php to read real quotes from published authors. 